Comment. Math. Helv. 74 (1999) 84-117

0010-2571/99/010084-34 \$1.50+0.20/0
(C) 1999 Birkhäuser Verlag, Basel

Commentarii Mathematici Helvetici

\title{
Spherical minimal immersions of the 3-sphere
}

\author{
Gabor Toth and Wolfgang Ziller*
}

\begin{abstract}
In 1966 Takahashi [11] proved that a minimal isometric immersion $f: S^{m}(1) \rightarrow$ $S^{N}(r)$ of round spheres exists iff $r=\sqrt{m / \lambda_{p}}$, where $\lambda_{p}$ is the $p$-th eigenvalue of the Laplacian on $S^{m}$; in this case, the components of $f$ are spherical harmonics on $S^{m}$ of order $p$. This immersion is unique up to congruence on the range and agrees with the generalized Veronese map if $m=2$ as was shown in 1967 by Calabi [1]. In 1971 DoCarmo and Wallach [3] proved that the same rigidity holds for $p=2,3$. The main aim of their work, however, was to show that, for $m \geq 3$ and $p \geq 4$, unicity fails, and, indeed, the set of (congruence classes of) minimal isometric immersions $f: S^{m} \rightarrow S^{N}\left(\sqrt{m / \lambda_{p}}\right)$ can be parametrized by a moduli space $\mathcal{M}_{m}^{p}$, a compact convex body in a representation space $\mathcal{F}_{m}^{p}$ of $S O(m+1)$ of dimension $\geq 18$. In 1994, the first author [14] determined the exact dimension of the moduli, and with Gauchman [5] in 1996, revealed intricate connections beween the irreducible components of $\mathcal{F}_{m}^{p}$ and the geometry of the immersions these components represent. The purpose of the present paper is to provide a complete geometric description of the fine details of the (boundary of the) 18-dimensional space $\mathcal{M}_{3}^{4}$, the first nontrivial moduli. This is made possible by several reductions that make use of the splitting $S O(4)=S U(2) \cdot S U(2)^{\prime}$ as well as rely on the structure of $S U(2)$ equivariant minimal isometric immersions treated in the work of DeTurck and the second author [2] in 1992. The equivariant imbedding theorem [14] asserts that the structure of $\mathcal{M}_{3}^{4}$ reappears in the moduli $\mathcal{M}_{m}^{p}$ for $m \geq 3$ and $p \geq 4$.
\end{abstract}

Mathematics Subject Classification (1991). Primary 53C42.

Keywords. Spherical minimal immersion, special unitary group.

Minimal isometric immersions of round spheres into round spheres form an interesting subject that has been studied by a number of authors, see e.g. [2,3,11,12]. We can write such maps either as isometric minimal immersions $f: S^{m}(1) \rightarrow$ $S^{N}(r)$ as was done in [2], or as we do here and as was done in [12], as minimal immersions $f: S^{m}(1) \rightarrow S^{N}(1)$ with homothety $1 / r^{2}$ (which we call spherical minimal immersions). The components of such an immersion must be eigenfunctions of the Laplacian on $S^{m}(1)$ which are hence harmonic homogeneous polynomials of degree $p$ with eigenvalue $\lambda_{p}=p(p+m-1)$, in which case the homothety is equal to $\lambda_{p} / m$. As is well known, if $m=2$, or if $m \geq 3$ and $p=2,3$, the immersion is unique up to congruence and agrees with the generalized Veronese immersion. But for $m \geq 3$ and $p \geq 4$, there are many such immersions, and for a fixed degree $p$ the

*With partial support from the National Science Foundation. 
congruence classes of such minimal immersions form a convex compact body in a vector space of high dimension. The dimension of this convex body was computed explicitly in [14]. In particular, if $m=3$ and $p=4$, it is equal to 18 (see also [9] for this special case) and the dimension quickly grows with $m$ and $p$.

The main purpose of the present paper is to examine this convex body in detail in the special case of $m=3, p=4$, although some of the results also apply to the general case. In this special case we are able to describe the structure of the convex body and its boundary completely with a number of interesting consequences. This is due to the fact that this low degree is the only case where the full convex body can be reconstructed in a certain way from the special subclass of equivariant minimal isometric immersions studied in more detail in $[2,5,6]$. This structure is also important for higher degrees and higher domain dimensions, since, according to the equivariant embedding theorem [14], the moduli space for degree four and domain dimension three can be equivariantly embedded into that for any degree $\geq 4$ and domain dimension $\geq 3$. In particular, using the examples developed here and in [2], a variety of spherical minimal immersions can be explicitly constructed from any domain of dimension $\geq 4$ and any degree $\geq 3$.

Before we describe our results, we set up some notation. If $f$ is a spherical minimal immersion $f: S^{m}(1) \rightarrow S^{N}(1) \subset \mathbf{R}^{N+1}=V$ which uses degree $p$ homogeneous harmonic polynomials, we denote by $\mathcal{M}_{f}$ the set of all spherical minimal immersions $f^{\prime}=A \circ f$ with $A: \mathbf{R}^{N+1} \rightarrow \mathbf{R}^{N^{\prime}+1}$ any linear map (in other words, the components of $f^{\prime} \in \mathcal{M}_{f}$ are linear combinations of the components of $f$ ). $\mathcal{M}_{f}$ is a compact convex body in a linear subspace $\mathcal{F}_{f} \subset S^{2} V$ parametrized by $A^{\top} A-I$. The points on the boundary of $\mathcal{M}_{f}$ consist of spherical minimal immersions with ambient dimension less than $N$. If $f_{p}$ is the standard spherical minimal immersion of $S^{m}$, consisting, up to homothety, of an orthonormal basis of the set $\mathcal{H}^{p}$ of all homogeneous harmonic polynomials of degree $p$, then $\mathcal{M}_{f_{p}}=\mathcal{M}^{p}$ is by definition the set of all spherical minimal immersions of degree $p$. For $f \in \mathcal{M}^{p}, \mathcal{M}_{f}$ forms a linear slice in $\mathcal{M}^{p}$. If $f$ lies in the interior of $\mathcal{M}^{p}, \mathcal{M}_{f}$ is of course equal to $\mathcal{M}^{p}$. But if $f$ lies on the boundary of $\mathcal{M}^{p}$, then $\mathcal{M}_{f}$ is a linear slice contained in the boundary of $\mathcal{M}^{p}$. We call $f$ linearly rigid if $\mathcal{M}_{f}=\{f\}$. The linearly rigid spherical minimal immersions are precisely the extremal points of $\mathcal{M}^{p}$ in the sense of convex geometry (by the connecting lemma in Section 1.1). By the Krein-Milman theorem, a convex set is the convex hull of its subset of extremal points, so that $\mathcal{M}^{p}$ is the convex hull of the linearly rigid spherical minimal immersions.

$S O(m+1)$ acts on $\mathcal{M}^{p}$ via precomposition $f \rightarrow f \circ A, f \in \mathcal{M}^{p}, A \in S O(m+1)$, which makes $\mathcal{F}^{p}$ a linear $S O(m+1)$ representation space. A group $G$ is the isotropy group of that action at a point $f$ iff $f$ is equivariant under $G$. We will say that $f$ is full if the image of $f$ spans all of $V$.

What is special about $m=3$ is that $S O(4)=S U(2) \cdot S U(2)^{\prime}$ with each $S U(2)$ acting transitively on $S^{3}$. Hence we can consider the $S U(2)$ and $S U(2)^{\prime}$ equivariant spherical minimal immersions, which can also be viewed as the fixed point set $\left(\mathcal{M}^{p}\right)^{S U(2)}$ or $\left(\mathcal{M}^{p}\right)^{S U(2)^{\prime}}$. We will show that for $p=4$, and this is what makes 
$m=3, p=4$ so special, that $\mathcal{F}^{4}=\left(\mathcal{F}^{4}\right)^{S U(2)} \oplus\left(\mathcal{F}^{4}\right)^{S U(2)^{\prime}}$, each one being a 9 dimensional linear subspace of $\mathcal{F}^{4}$. As an $S O(4)$ representation, this splitting is $R_{8}^{\prime} \oplus R_{8}$ where $S U(2)$ acts trivially on $R_{8}^{\prime}$ and via the unique 9 dimensional irreducible representation on $R_{8}$ and in a reversed role for $S U(2)^{\prime}$. Furthermore, we show that each point in $\partial \mathcal{M}^{4}$ lies on a straight line in $\mathcal{F}^{4}$ connecting a point $f_{1} \in \partial\left(\mathcal{M}^{4}\right)^{S U(2)}$ with a point $f_{2} \in \partial\left(\mathcal{M}^{4}\right)^{S U(2)^{\prime}}$. This straight line consists of the immersions $\left(\sqrt{c_{1}} f_{1}, \sqrt{c_{2}} f_{2}\right)$ with $c_{1}>0, c_{2}>0, c_{1}+c_{2}=1$. An orientation reversing isometry of $S^{3}$ will interchange $R_{8}^{\prime}$ and $R_{8}$ and hence $\partial\left(\mathcal{M}^{4}\right)^{S U(2)}$ with $\partial\left(\mathcal{M}^{4}\right)^{S U(2)^{\prime}}$. Thus the boundary immersions $\partial \mathcal{M}^{4}$ are completely determined by the equivariant boundary immersions in $\partial\left(\mathcal{M}^{4}\right)^{S U(2)}$. Equivariant immersions in $\left(\mathcal{M}^{4}\right)^{S U(2)}$ have ambient dimension $N+1=5,10,15,20$ or 25 , and one can easily exclude $N+1=5$. This will enable us to completely determine all ambient dimensions:

Theorem A. If $f: S^{3} \rightarrow S^{N}$ is a full degree 4 spherical minimal immersion, then the only possible ambient dimensions are $N+1=10,15,16$, or $19-25$, and each one occurs.

Furthermore, it will follow that the spherical minimal immersions with $N+1=$ 10,15 , or 20 consist only of $S U(2)$ or $S U(2)^{\prime}$ equivariant ones. Combining this with Proposition 1 in [2], p.449, yields the following uniqueness result for the lowest possible ambient dimension:

Theorem B. There exists a degree 4 spherical minimal immersion $\mathcal{I}: S^{3} \rightarrow S^{9}$ such that if $f: S^{3} \rightarrow S^{9}$ is any degree 4 spherical minimal immersion, then there exist isometries $A \in O(10)$ and $B \in O(4)$ such that $f=A \circ \mathcal{I} \circ B$. Furthermore $\mathcal{I}$ is $S U(2)$ equivariant with image an embedded space form $S^{3} / D_{2}^{*}$, where $D_{2}^{*}$ is the quaternion group $\{ \pm 1, \pm i, \pm j, \pm k\}$.

One can easily describe $\mathcal{I}: S^{3} \rightarrow S^{9}$ explicitly:

$$
\begin{gathered}
\mathcal{I}(z, w)=\left((1 / \sqrt{2})\left(z^{4}-\bar{w}^{4}\right), \sqrt{6} z^{2} \bar{w}^{2}, \sqrt{2}\left(z^{3} w+\bar{z} \bar{w}^{3}\right), \sqrt{6}\left(z \bar{z}^{2} w-\bar{z} w^{2} \bar{w}\right),\right. \\
\left.\sqrt{3 / 2}\left(z^{2} w^{2}-\bar{z}^{2} \bar{w}^{2}\right),(1 / \sqrt{2})\left(|z|^{4}-4|z|^{2}|w|^{2}+|w|^{4}\right)\right) .
\end{gathered}
$$

Here $z$ and $w$ are complex with $|z|^{2}+|w|^{2}=1$. Notice that the first four coordinates are complex, the fifth is purely imaginary and the sixth is real so that $\mathcal{I}$ maps into $\mathbf{R}^{10}$ and one easily checks that it maps into the unit sphere in $\mathbf{R}^{10}$. Also notice that the map is invariant under $(z, w) \rightarrow(i z, i w)$ and $(z, w) \rightarrow(\sqrt{i} \bar{w},-\sqrt{i} \bar{z})$ which generate a group isomorphic to $D_{2}^{*}$. It will follow that $\mathcal{I}$ is an embedding of $S^{3} / D_{2}^{*}$ into $S^{9}$. 
Finally we describe the equivariant boundary immersions in $\left(\mathcal{M}^{4}\right)^{S U(2)} \subset \mathcal{F}^{4}$. They naturally divide into 3 subsets, denoted by I, II, and III, depending on if the ambient dimension is $N+1=10,15$, or 20 . Notice that the action of $S O(4)$ reduces to an $S U(2)^{\prime}$ action on $\left(\mathcal{M}^{4}\right)^{S U(2)}$ and hence each set is the union of $S U(2)^{\prime}$ orbits. We will show:

Theorem C. $\partial\left(\mathcal{M}^{4}\right)^{S U(2)}=\mathbf{I} \cup \mathbf{I I} \cup \mathbf{I I I}$ and one has:

a) $\mathbf{I}$ is a single $S U(2)^{\prime}$ orbit (the $S U(2)^{\prime}$ orbit of $\mathcal{I}$ ) and is an octahedral manifold $S^{3} / \mathbf{O}^{*}$ embedded minimally in a sphere in $R_{8}^{\prime}=\left(\mathcal{F}^{4}\right)^{S U(2)}$, the embedding given by a degree 8 equivariant minimal isometric immersion.

b) $\mathbf{I I}=\mathbf{I I}_{0} \cup \mathbf{I I}_{1}$ where $\mathbf{I I}_{0}$ is a 6 dimensional connected set consisting of linearly rigid immersions, and $\mathbf{I I}_{1}$ is the 4 dimensional set $S U(2)^{\prime} \cdot \operatorname{int} \mathcal{D}$ where $\mathcal{D}$ is a flat 2 -dimensional disk with boundary circle on the octahedral manifold $\mathbf{I}$. Furthermore $\mathcal{D}=\mathcal{M}_{\mathcal{J}}$, where $\mathcal{J}$ is the unique $U(2)$ equivariant minimal immersion in $\mathbf{I I}$.

c) III is dense, open and connected in the 8 dimensional boundary $\partial\left(\mathcal{M}^{4}\right)^{S U(2)}$. The opposite $\mathcal{J}^{o}$ of $\mathcal{J}$ on the boundary $\partial\left(\mathcal{M}^{4}\right)^{S U(2)}$ is of type III. $\mathcal{M}_{\mathcal{J}}$ is 6 dimensional and

$$
\mathbf{I I I}=S U(2)^{\prime} \cdot \operatorname{int} \mathcal{M}_{\mathcal{J}_{o}}
$$

The $U(2)$ equivariant map $\mathcal{J}: S^{3} \rightarrow S^{14}$, is given explicitly as follows:

$$
\begin{aligned}
& \mathcal{J}(z, w)=(1 / \sqrt{2})\left(z^{4}, w^{4}, 2 \sqrt{3} z^{2} \bar{w}^{2}, 2 z^{3} w, 2 z w^{3}\right. \\
& \left.2 \sqrt{3}\left(z \bar{z}^{2} w-\bar{z} w^{2} \bar{w}\right), \sqrt{6} z^{2} w^{2},|z|^{4}-4|z|^{2}|w|^{2}+|w|^{4}\right) .
\end{aligned}
$$

$\mathcal{J}$ is invariant under $(z, w) \rightarrow(i z, i w)$ and the image is an embedded lens space $S^{3} / \mathbf{Z}_{4} . \mathcal{J}$ is at the center of the disk $\mathcal{D}=\mathcal{M}_{\mathcal{J}}$ and the center of $U(2)$ acts as a rotation on this disk and hence $S U(2)^{\prime} \cdot \mathcal{D}=S U(2)^{\prime} \cdot \mathcal{M}_{\mathcal{J}}$ is 4-dimensional. This gives an explicit description to all elements in $\mathbf{I I}_{1}$.

There exists only a 1-dimensional space of $U(2)$ equivariant immersions in $\left(\mathcal{M}^{4}\right)^{S U(2)}$ and hence only two $U(2)$ equivariant elements in $\partial\left(\mathcal{M}^{4}\right)^{S U(2)}$, one of which is $\mathcal{J}$ and the other one the antipodal point $\mathcal{J}_{o}$ (which lies in III). The orbit $S U(2)^{\prime} \mathcal{J}$ (and hence also $S U(2)^{\prime} \mathcal{J}_{o}$ ) is a minimally embedded $\mathbf{R} P^{2}$ in a sphere in $R_{8}^{\prime}$ which is in fact the standard rigid minimal isometric immersion for $m=2$ and $p=4$.

It is more difficult to find explicit examples in the 6-dimensional set of linearly rigid elements in $\mathbf{I I}_{0}$. One explicit example in $\mathbf{I I}_{0}$ is $\mathcal{I}_{o}$, the antipodal to the immersion $\mathcal{I}$ in Theorem B. Since the components of $\mathcal{I}$ are orthogonal of equal length, one can easily write down $\mathcal{I}_{o}$ explicitly by choosing such a basis for the orthogonal complement of the subspace spanned by the components of $\mathcal{I}$ in $\mathcal{H}^{4}$. In particular $\mathcal{I}_{o}: S^{3} \rightarrow S^{14}$ and we will show that $\mathcal{I}_{o}$ is linearly rigid. 
By Theorem C, the only exremal points in $\left(\mathcal{M}^{4}\right)^{S U(2)}$ are the elements in $\mathbf{I}$ and $\mathbf{I I}_{0}$. Hence $\left(\mathcal{M}^{4}\right)^{S U(2)}$ is the convex hull of such immersions. As a consequence, all of $\mathcal{M}^{4}$ is the convex hull of $\mathbf{I}, \mathbf{I I}_{0}$ and the images $\mathbf{I}^{\prime}, \mathbf{I} \mathbf{I}_{0}^{\prime}$ in $\partial\left(\mathcal{M}^{4}\right)^{S U(2)^{\prime}}$

Also, notice that if we connect a linear slice $\mathcal{M}_{f}$ in III with a corresponding linear slice in $\partial\left(\mathcal{M}^{4}\right)^{S U(2)^{\prime}}$, we obtain a 13 dimensional linear slice in $\partial \mathcal{M}^{4}$, which is the largest dimensional linear slice in the 17-dimensional boundary.

Another interesting question that one can ask, is about the image $f\left(S^{3}\right)$. Are they embeddings or immersions of a quotient $S^{3} / G$ and what quotients can arise? For interior points of $\mathcal{M}^{p}$, it was observed in [18] that they are always embedded spheres or projective spaces, depending on if $p$ is odd or even. But on $\partial \mathcal{M}^{p}$ many other images can occur. It is still an open problem whether all space forms can arise. In [2] it was shown that every homogeneous space form admits an equivariant minimal isometric embedding and in [4] an example was constructed of an inhomogeneous lens space with a minimal isometric immersion into $S^{190}$ with degree 32 polynomials. It follows from [2] that for the equivariant maps in $\mathcal{M}^{4}$, the images are embedded homogeneous space forms $S^{3} / D_{2}^{*}$, lens spaces $S^{3} / Z_{4}$, or projective spaces (the generic case). It also follows that there exist many non equivariant minimal isometric embeddings of the homogeneous space forms $S^{3} / D_{2}^{*}$ and $S^{3} / Z_{4}$.

For $m=3$ and $p>4$ there are corresponding results, but they only describe a portion of the moduli space which are not sufficient to prove analogues of Theorems $\mathrm{A}$ and B. See Theorems 4 and 5 for details. This is due to the fact that $\mathcal{F}^{p}$ splits into many irreducible summands under $S O(4)$ (see Theorem 3) and our methods apply to only some of those summands.

It is interesting to compare the results in this paper with some of the results in [15]. One can formulate the above mentioned connecting lemma, by saying that $\mathcal{M}^{4}$ is the convex hull of its slices by the two irreducible components in $\mathcal{F}^{4}$. For $p=6$ (and $m=3$ ) the moduli space of $S U(2)$ equivariant immersions $\left(\mathcal{M}^{6}\right)^{S U(2)}$ has two irreducible components and in [15] it is shown that a minimal immersion in each component has ambient dimension $N+1 \geq 14$. The degree 6 minimal immersion $f: S^{3} \rightarrow S^{6}$ constructed in [2] cannot lie in either component and not in the convex hull of the linear slices with each component either. Thus for larger degrees, the moduli space is not any more the convex hull of its linear irreducible slices, which shows that the structure of the moduli space gets much more complicated for $p>4$.

In section 1 we collect several preparatory results that hold in the general case. Section 2 describes some results about the $S U(2)$ equivariant minimal maps of $S^{3}$ but any degree $p$ and in section 3 we prove the above results for $m=3$ and $p=4$. 


\section{General theory}

\subsection{Eigenmaps}

A spherical harmonic of order $p$ on $S^{m}$ is, by definition, an eigenfunction of the Laplacian $\triangle$ on $S^{m}$ with eigenvalue $\lambda_{p}=p(p+m-1)$, or equivalently, the restriction (to $S^{m}$ ) of a harmonic homogeneous polynomial in the variables $\left(x_{0}, \ldots, x_{m}\right) \in \mathbf{R}^{m+1}$. The linear space of spherical harmonics is denoted by $\mathcal{H}^{p}=\mathcal{H}_{m}^{p}$. (Unless important, we suppress the source dimension $m$.) In a similar vein, a $p$-eigenmap $f: S^{m} \rightarrow S_{V}$, where $V$ is a Euclidean vector space with $S_{V}$, the unit sphere in $V$, is a map whose components $\alpha \circ f, \alpha \in V^{*}$, are spherical harmonics of order $p$ on $S^{m}$, or equivalently, a harmonic $p$-homogeneous spherical polynomial map $f: \mathbf{R}^{m+1} \rightarrow V$. Spherical means that $f$ maps $S^{m}$ into $S_{V}$ and, in this case, we identify $f: \mathbf{R}^{m+1} \rightarrow V$ with its restriction $f: S^{m} \rightarrow S_{V}$.

$f: S^{m} \rightarrow S_{V}$ is full if it has no zero component. In this case, precomposition with $f, \alpha \rightarrow \alpha \circ f, \alpha \in V^{*}$, gives a linear embedding $V^{*} \rightarrow \mathcal{H}^{p}$ whose image; the space of components of $f$, is denoted by $V_{f}$. Since $V$ is Euclidean, we have the isomorphisms

$$
V \cong V^{*} \cong V_{f} \subset \mathcal{H}^{p}
$$

In what follows, $V, V^{*}$ and $V_{f}$ will be identified under these isomorphisms.

Two $p$-eigenmaps $f_{1}: S^{m} \rightarrow S_{V_{1}}$ and $f_{2}: S^{m} \rightarrow S_{V_{2}}$ are said to be congruent if $f_{2}=U \circ f_{1}$ for some isometry $U: V_{1} \rightarrow V_{2}$.

Let $f: S^{m} \rightarrow S_{V}$ and $f^{\prime}: S^{m} \rightarrow S_{V^{\prime}}$ be full $p$-eigenmaps. $f^{\prime}$ is said to be derived from $f$, written as $f^{\prime} \leftarrow f$, if there exists a linear map $A: V \rightarrow V^{\prime}$ such that $A \circ f=f^{\prime}$. Since $f$ is full, $A$ is uniquely determined. Since $f^{\prime}$ is also full, $A$ is onto.

Let $f: S^{m} \rightarrow S_{V}$ be a full $p$-eigenmap. Let $\mathcal{E}_{f} \subset S^{2} V$ denote the orthogonal complement of

$$
\mathcal{W}_{f}=\operatorname{span}\left\{f(x) \odot f(x) \mid x \in S^{m}\right\}
$$

in $S^{2} V$. Let

$$
\mathcal{L}_{f}=\left\{C \in \mathcal{E}_{f} \mid C+I \geq 0\right\}
$$

where $I=I_{V}=$ identity of $V$ and ' $\geq$ ' means 'positive semidefinite'. Clearly, $\mathcal{L}_{f}$ is a convex body in $\mathcal{E}_{f}$ and the origin of $\mathcal{E}_{f}$ is contained in the interior of $\mathcal{L}_{f}$. A DoCarmo-Wallach type argument gives the following:

Theorem 1. Given a full p-eigenmap $f: S^{m} \rightarrow S_{V}$, the set of congruence classes of full eigenmaps $f^{\prime}: S^{m} \rightarrow S_{V^{\prime}}$ that are derived from $f$ can be parametrized by the convex body $\mathcal{L}_{f}$. The parametrization is given by associating to the congruence class of $f^{\prime}$ the endomorphism

$$
\left\langle f^{\prime}\right\rangle_{f}=A^{\top} \cdot A-I \in S^{2} V
$$

where $f^{\prime}=A \circ f$ and $^{\top}$ denotes transpose. 
Remark. For (all) spherical minimal immersions this is due to DoCarmo-Wallach $[3,17]$. The present relative version is taken from [12].

The convex body $\mathcal{L}_{f}$ of $\mathcal{E}_{f}\left(\subset S^{2} V\right)$ is called the moduli space associated to the full $p$-eigenmap $f: S^{m} \rightarrow S_{V}$. $f$ corresponds to the origin $0\left(=\langle f\rangle_{f}\right)$. The interior int $\mathcal{L}_{f}$ parametrizes those full $p$-eigenmaps $f^{\prime}: S^{m} \rightarrow S_{V^{\prime}}$ for which $f \leftarrow f^{\prime}$, or equivalently, $f^{\prime}=A \circ f$ with $A: V \rightarrow V^{\prime}$ invertible. As $A$ is onto, this holds iff $\operatorname{dim} V=\operatorname{dim} V^{\prime}$. Thus the boundary $\partial \mathcal{L}_{f}$ corresponds to those $p$-eigenmaps $f^{\prime}: S^{m} \rightarrow S_{V^{\prime}}$ for which $f^{\prime}<f$ and $\operatorname{dim} V^{\prime}<\operatorname{dim} V$.

Since $\angle$ is a transitive relation, for $f^{\prime}<f, \mathcal{L}_{f^{\prime}}$ can be embedded into $\mathcal{L}_{f}$. The image is an affine slice (that is, the intersection of $\mathcal{L}_{f}$ with an affine subspace). More precisely, let $f: S^{m} \rightarrow S_{V}$ and $f^{\prime}: S^{m} \rightarrow S_{V^{\prime}}$ with $f^{\prime} \leftarrow f$, i.e. $f^{\prime}=A \circ f$, and define

$$
\iota: S^{2} V^{\prime} \rightarrow S^{2} V
$$

by

$$
\iota\left(C^{\prime}\right)=A^{\top} \cdot C^{\prime} \cdot A+\left\langle f^{\prime}\right\rangle_{f}=A^{\top} \cdot\left(C^{\prime}+I_{V^{\prime}}\right) \cdot A-I_{V} .
$$

Then, as easy computation shows, $\iota$ maps $\mathcal{E}_{f^{\prime}}$ injectively into $\mathcal{E}_{f}$ and

$$
\iota\left(\mathcal{L}_{f^{\prime}}\right)=\iota\left(\mathcal{E}_{f^{\prime}}\right) \cap \mathcal{L}_{f} .
$$

From now on, we identify $\mathcal{L}_{f^{\prime}}$ with its image in $\mathcal{L}_{f}$.

Up to scaling the components of an orthonormal basis in $\mathcal{H}^{p}$ with respect to the $L^{2}$-scalar product give rise to the standard $p$-eigenmap $f_{p}: S^{m} \rightarrow S_{\mathcal{H}^{p}}$. For $f=f_{p}$, we denote $\mathcal{L}^{p}=\mathcal{L}_{m}^{p}=\mathcal{L}_{f_{p}}, \mathcal{E}^{p}=\mathcal{E}_{m}^{p}=\mathcal{E}_{f_{p}}$ etc. We call $\mathcal{L}^{p}$ the standard moduli space. Since all $p$-eigenmaps are derived from $f_{p}, \mathcal{L}^{p}$ parametrizes the congruence classes of all full $p$-eigenmaps $f: S^{m} \rightarrow S_{V}$. For simplicity, we set $\langle f\rangle_{f_{p}}=\langle f\rangle$. We say that a full p-eigenmap $f: S^{m} \rightarrow S_{V}$ is of boundary type if $\operatorname{dim} V<\operatorname{dim} \mathcal{H}^{p}$, or equivalently, if $\langle f\rangle \in \partial \mathcal{L}^{p}$. Also, for any full $p$-eigenmap $f: S^{m} \rightarrow S_{V}, \mathcal{L}_{f}$ is an affine slice of $\mathcal{L}^{p}$. Integrating the condition of sphericality for $f$, we see $[3,17]$ that $\mathcal{E}^{p}$ consists of traceless endomorphisms of $S^{2}\left(\mathcal{H}^{p}\right)$. We thus have:

Corollary. $\mathcal{L}^{p}$ and (hence) $\mathcal{L}_{f}$ are compact.

Let $f: S^{m} \rightarrow S_{V}$ and $f^{\prime}: S^{m} \rightarrow S_{V^{\prime}}$ be full $p$-eigenmaps and assume that $f^{\prime}<f$ with $f^{\prime}=A \circ f$, where $A: V \rightarrow V^{\prime}$ is linear and onto. Then $V_{f^{\prime}}$ is contained in $V_{f}$ and the inclusion $V_{f^{\prime}} \subset V_{f}$ is given by sending $\alpha^{\prime} \circ f^{\prime}, \alpha^{\prime} \in\left(V^{\prime}\right)^{*}$, to $\left(\alpha^{\prime} \circ A\right) \circ f$. Connecting Lemma. Let $f_{1}: S^{m} \rightarrow S_{V_{1}}$ and $f_{2}: S^{m} \rightarrow S_{V_{2}}$ be full p-eigenmaps and assume that they are incongruent. Let $c_{1}, c_{2}>0$ with $c_{1}+c_{2}=1$. Then the point

$$
c_{1}\left\langle f_{1}\right\rangle+c_{2}\left\langle f_{2}\right\rangle \in \mathcal{L}^{p}
$$

on the segment connecting $\left\langle f_{1}\right\rangle$ and $\left\langle f_{2}\right\rangle$ is represented by the p-eigenmap $f: S^{m} \rightarrow$ $S_{V}, V=V_{1} \times V_{2}$, defined by $f=\left(\sqrt{c_{1}} f_{1}, \sqrt{c_{2}} f_{2}\right)$ and made full. In particular, 
$f \rightarrow f_{1}, f_{2}$ and

$$
V_{f}=V_{f_{1}}+V_{f_{2}}
$$

so that

$$
\operatorname{dim} V_{f}=\operatorname{dim} V_{f_{1}}+\operatorname{dim} V_{f_{2}}-\operatorname{dim}\left(V_{f_{1}} \bigcap V_{f_{2}}\right) .
$$

Proof. Setting $f_{1}=A_{1} \circ f_{p}$ and $f_{2}=A_{2} \circ f_{p}$ with $A_{1}: \mathcal{H}^{p} \rightarrow V_{1}$ and $A_{2}: \mathcal{H}^{p} \rightarrow V_{2}$ linear and onto, we have

$$
\left\langle f_{1}\right\rangle=A_{1}^{\top} A_{1}-I \quad \text { and } \quad\left\langle f_{2}\right\rangle=A_{2}^{\top} A_{2}-I .
$$

By definition, $f=\left(\sqrt{c_{1}} A_{1}, \sqrt{c_{2}} A_{2}\right) \circ f_{p}$ so that

$$
\begin{aligned}
\langle f\rangle & =\left(c_{1} A_{1}^{\top} A_{1}+c_{2} A_{2}^{\top} A_{2}\right)-I \\
& =c_{1}\left(A_{1}^{\top} A_{1}-I\right)+c_{2}\left(A_{2}^{\top} A_{2}-I\right) \\
& =c_{1}\left\langle f_{1}\right\rangle+c_{2}\left\langle f_{2}\right\rangle
\end{aligned}
$$

since $c_{1}+c_{2}=1$. The rest is clear.

Given a full $p$-eigenmap $f: S^{m} \rightarrow S_{V}$ of boundary type, $\langle f\rangle \in \partial \mathcal{L}^{p}$, the line $\mathbf{R} \cdot\langle f\rangle$ intersects $\partial \mathcal{L}^{p}$ in $\langle f\rangle$ and another point called the antipodal of $\langle f\rangle$. A representative $f_{o}: S^{m} \rightarrow S_{V_{o}}$ of the antipodal of $\langle f\rangle$ is called the antipodal $p$-eigenmap of $f$. ( $f_{o}$ is unique up to congruence.)

The connection between $f$ and $f_{o}$ is subtle. A related (and again subtle) problem has been posed by R.T.Smith in his Thesis [10]: Given a $p$-eigenmap $f: S^{m} \rightarrow S_{V}$, does there exist a $p$-eigenmap $f^{\prime}: S^{m} \rightarrow S_{V^{\prime}}$ such that $V_{f^{\prime}}$ is the orthogonal complement of $V_{f}$ in $\mathcal{H}^{p}$ ? The following observation will be useful:

Antipodal Lemma. Let $f: S^{m} \rightarrow S_{V}$ be a full p-eigenmap of boundary type and assume that, relative to an orthonormal basis in $V$, the components of $f$ are orthogonal in $\mathcal{H}^{p}$ and have the same norm. Then the antipodal $f_{o}: S^{m} \rightarrow S_{V_{o}}$ of $f$ has the same property and

$$
V_{f} \oplus V_{f_{o}}=\mathcal{H}^{p}
$$

is an orthogonal direct sum.

Proof. Relative to an orthonormal basis in $V$ and up to a constant multiple, the components of $f$ give an orthonormal basis $\mathcal{V}$ in $V_{f} \subset \mathcal{H}^{p}$. Select an orthonormal basis $\mathcal{V}_{o}$ from $V_{f}^{\perp} \subset \mathcal{H}^{p}$. Let $f_{o}: \mathbf{R}^{m+1} \rightarrow V_{o}$ be a full harmonic $p$-homogeneous polynomial map whose components, relative to an orthonormal basis in $V_{o}$, are the elements of $\mathcal{V}_{o}$. Since $\mathcal{V} \cup \mathcal{V}_{o}$ (suitably normalized) gives the components of $f_{p}$, up to a constant multiple, $f_{o}$ is spherical so that it restricts to a full $p$-eigenmap $f_{o}: S^{m} \rightarrow S_{V_{o}}$. By construction, $f_{p}=\left(c f, c_{o} f_{o}\right)$ for some constants $c, c_{o}>0$. Taking norms, we have $c^{2}+c_{o}^{2}=1$ so that the connecting lemma applies. We obtain that the origin is on the segment connecting $\langle f\rangle$ and $\left\langle f_{o}\right\rangle$. Since both $f$ and $f_{o}$ are of boundary type, $f_{o}$ is the antipodal of $f$. 
Let $f: S^{m} \rightarrow S_{V}$ be a $p$-eigenmap and assume that $f$ is equivariant with respect to a homomorphism $\rho_{f}: G \rightarrow S O(V)$, where $G \subset S O(m+1)$ is a closed subgroup. Equivariance means that

$$
f \circ g=\rho_{f}(g) \circ f,
$$

for all $g \in G$. $\rho_{f}$ defines an orthogonal $G$-module structure on $V$ and therefore on $V^{*}$. Under the isomorphism $V^{*} \cong V_{f} \subset \mathcal{H}^{p}, V$ becomes a $G$-submodule of $\left.\mathcal{H}^{p}\right|_{G}$. Indeed, since $g \in G$ acts on $V^{*}$ as $g \cdot \alpha=\alpha \circ \rho_{f}(g)^{-1}, \alpha \in V^{*}$, we have

$$
(g \cdot \alpha) \circ f=\left(\alpha \circ \rho_{f}(g)^{-1}\right) \circ f=(\alpha \circ f) \circ g^{-1} .
$$

The $G$-module structure on $V$ extends to that of $S^{2} V$ given by

$$
g \cdot C=\rho_{f}(g) \cdot C \cdot \rho_{f}(g)^{-1} .
$$

$\mathcal{E}_{f}$ is clearly a $G$-submodule of $S^{2} V$. In fact, $\mathcal{L}_{f}$ is $G$-invariant since, for $f^{\prime} \leftarrow f$, we have

$$
g \cdot\left\langle f^{\prime}\right\rangle_{f}=\left\langle f^{\prime} \circ g^{-1}\right\rangle_{f}
$$

Given a closed subgroup $G^{\prime} \subset G$, a $p$-eigenmap $f^{\prime}: S^{m} \rightarrow S_{V^{\prime}}, f^{\prime}<f$, is equivariant with respect to a homomorphism $\rho_{f^{\prime}}: G^{\prime} \rightarrow S O\left(V^{\prime}\right)$ iff $\left\langle f^{\prime}\right\rangle_{f}$ is left fixed by $G^{\prime}$. Thus the congruence classes of full $p$-eigenmaps that are equivariant with group $G^{\prime}$ are parametrized by the linear slice

$$
\left(\mathcal{L}_{f}\right)^{G^{\prime}}=\mathcal{L}_{f} \cap\left(\mathcal{E}_{f}\right)^{G^{\prime}} .
$$

Remark. Let $f: S^{m} \rightarrow S_{V}$ be a full $p$-eigenmap and assume that, relative to an orthonormal basis of $V$, the components of $f$ in $\mathcal{H}^{p}$ form an orthogonal basis $\mathcal{V}$ with the same norm. Then, the isotropy group at $\langle f\rangle \in \mathcal{L}^{p}$ can be written as

$$
S O(m+1)_{\langle f\rangle}=\left\{g \in S O(m+1) \mid V_{f}=V_{f \circ g}\right\} .
$$

Indeed, for $g \in S O(m+1), U \in S O(V)$ with $U \circ f=f \circ g$ exists iff $V_{f}=V_{f \circ g}$ and is the transfer matrix between $\mathcal{V}$ and $\mathcal{V} \circ g$ obtained by precomposing the elements of $\mathcal{V}$ with $g$.

The following lemma is contained in [12] (pp.24-25).

Transversality Lemma. Let $f: S^{m} \rightarrow S_{V}$ be a full p-eigenmap that is equivariant with respect to a homomorphism $\rho_{f}: G \rightarrow S O(V)$, where $G \subset S O(m+1)$ is a closed subgroup. Let $f^{\prime}: S^{m} \rightarrow S_{V^{\prime}}$ be a full p-eigenmap such that $\left\langle f^{\prime}\right\rangle_{f} \in \operatorname{int} \mathcal{L}_{f}$. If $a: \mathbf{R} \rightarrow G$ is a 1-parameter subgroup such that the orbit $t \mapsto a(t) \cdot\left\langle f^{\prime}\right\rangle_{f}, t \in \mathbf{R}$, is tangent to int $\mathcal{L}_{f}$ at $t=0$ then it is entirely contained in $\operatorname{int} \mathcal{L}_{f}$.

The standard $p$-eigenmap $f_{p}: S^{m} \rightarrow S_{\mathcal{H}^{p}}$ is equivariant with respect to the homomorphism $\rho_{p}: S O(m+1) \rightarrow S O\left(\mathcal{H}^{p}\right)$ that is just the orthogonal $S O(m+1)$ module structure on $\mathcal{H}^{p}$ given by precomposing spherical harmonics by the inverse of linear isometries on $S^{m}$. Thus $\mathcal{E}^{p}$ is an $S O(m+1)$-submodule of $S^{2}\left(\mathcal{H}^{p}\right)$. A result of Calabi [1] asserts that $\mathcal{E}_{2}^{p}$ is trivial for any $p \geq 2$. As we will see below $\mathcal{E}_{m}^{p}$ is nontrivial for $m \geq 3$ and $p \geq 2$. 


\subsection{Spherical minimal immersions}

A spherical minimal immersion is a conformal p-eigenmap $f: S^{m} \rightarrow S_{V}$. The conformality factor is then $\lambda_{p} / m$. We say that $f: S^{m} \rightarrow S_{V}$ is a minimal immersion with homothety $\lambda_{p} / m$. The condition of homothety can be written as

$$
\left\langle f_{*}(X), f_{*}(Y)\right\rangle=\left(\lambda_{p} / m\right)\langle X, Y\rangle,
$$

for all vector fields $X$ and $Y$ on $S^{m}$.

Let $f: S^{m} \rightarrow S_{V}$ be a full homothetic minimal immersion with homothety $\lambda_{p} / m$. We define

$$
\mathcal{F}_{f}=\operatorname{span}\left\{f_{*}(X)^{\sim} \odot f_{*}(Y)^{\curlyvee} \mid X, Y \in T\left(S^{m}\right)\right\}^{\perp} \subset S^{2} V
$$

where denotes translation of vectors to the origin. (Here and in what follows it is understood that $X$ and $Y$ belong to the same tangent space of $S^{m}$.)

Let

$$
\mathcal{M}_{f}=\left\{C \in \mathcal{F}_{f} \mid C+I \geq 0\right\} .
$$

A result of Takahashi [11] implies that

$$
\mathcal{F}_{f} \subset \mathcal{E}_{f}
$$

(cf. [12]). The defining relation for $\mathcal{L}_{f}$ in $\mathcal{E}_{f}$ is the same as for $\mathcal{M}_{f}$ in $\mathcal{F}_{f}$. Thus, the inclusion above gives

$$
\mathcal{M}_{f}=\mathcal{F}_{f} \cap \mathcal{L}_{f}
$$

as a linear slice of $\mathcal{L}_{f}$. In particular, $\mathcal{M}_{f}$ is a compact convex body in $\mathcal{F}_{f}$.

Theorem 2. Given a full homothetic minimal immersion $f: S^{m} \rightarrow S_{V}$, the set of congruence classes of full homothetic minimal immersions $f^{\prime}: S^{m} \rightarrow S_{V^{\prime}}$ that are derived from $f$ can be parametrized by the convex body $\mathcal{M}_{f}$.

The convex body $\mathcal{M}_{f}$ is said to be the moduli space associated to the full minimal immersion $f: S^{m} \rightarrow S_{V}$. We say that $f$ is linearly rigid [17] if $\mathcal{M}_{f}$ reduces to a point. Note that the connecting, antipodal and transversality lemmas remain valid in the context of minimal immersions.

Since $S O(m+1)$ acts transitively on the unit sphere bundle of $S^{m}$, the standard $p$-eigenmap $f_{p}: S^{m} \rightarrow S_{\mathcal{H}^{p}}$ is conformal and thereby a minimal immersion with homothety $\lambda_{p} / m$. The standard moduli space $\mathcal{M}^{p}=\mathcal{M}_{m}^{p}=\mathcal{M}_{f_{p}}$ is the linear slice of $\mathcal{L}^{p}$ by the $S O(m+1)$-submodule $\mathcal{F}^{p}=\mathcal{F}_{m}^{p}=\mathcal{F}_{f_{p}} \subset S^{2}\left(\mathcal{H}^{p}\right)$ and it parametrizes the congruence classes of all full minimal immersions with homothety $\lambda_{p} / m$. The ultimate goal is to describe $\mathcal{M}^{p}$. As a first step, we need to determine the $S O(m+1)$-module structure of (its linear span) $\mathcal{F}^{p}$, that is, the decomposition of $\mathcal{F}^{p}$ into irreducible submodules. By DoCarmo-Wallach [3,17], $\mathcal{F}^{p}$ is nontrivial iff $m \geq 3$ and $p \geq 4$. 


\subsection{Some representation theory}

$V^{\left(u_{1}, \ldots, u_{d}\right)}=V_{m+1}^{\left(u_{1}, \ldots, u_{d}\right)}, d=[(m+1) / 2]$, denotes the (unique) complex irreducible $S O(m+1)$-module with highest weight vector $\left(u_{1}, \ldots, u_{d}\right)$ relative to the standard maximal torus in $S O(m+1)$. In particular, $V^{(p, 0, \ldots, 0)}=\mathcal{H}^{p}$ as complex $S O(m+1)$ modules. (Here and in what follows we denote $\mathcal{H}^{p}$ and its complexification by the same symbol.) For $m=3$ and $v>0, V_{4}^{(u, v)}$ means $V_{4}^{(u, v)} \oplus V_{4}^{(u,-v)}$.

By DoCarmo-Wallach [3]:

$$
\mathcal{H}^{p} \otimes \mathcal{H}^{q}=\sum_{(u, v) \in \triangle_{0}^{p, q} ; u+v \equiv p+q(\bmod 2)} V^{(u, v, 0, \ldots, 0)}, p \geq q \geq 1, m \geq 3,
$$

where $\triangle_{0}^{p, q}$ is the closed convex triangle in $\mathbf{R}^{2}$ with vertices $(p-q, 0),(p, q)$ and $(p+q, 0)$.

Setting $p=q$ and deleting the components that belong to the skew-symmetric part of $\mathcal{H}^{p} \otimes \mathcal{H}^{p}$, we have

$$
S^{2}\left(\mathcal{H}^{p}\right)=\sum_{(u, v) \in \triangle_{0}^{p} ; u, v \text { even }} V^{(u, v, 0, \ldots, 0)}
$$

where we simplified the notation by setting $\triangle_{0}^{p}=\triangle_{0}^{p, p}$.

We have

$$
\mathcal{E}^{p} \otimes \mathbf{C}=\sum_{(u, v) \in \triangle_{1}^{p} ; u, v \text { even }} V^{(u, v, 0, \ldots, 0)}
$$

where $\triangle_{1}^{p}$ is the closed convex triangle in $\mathbf{R}^{2}$ with vertices $(2,2),(p, p)$ and $(2 p-$ $2,2)$. For a quick proof cf. [5].

Remark. Let $f: S^{m} \rightarrow S_{V}$ be a full $p$-eigenmap and assume that

$$
\langle f\rangle \in V^{(2 p-2 l, 2 l, 0, \ldots, 0)}, \quad l=1, \ldots,[p / 2] .
$$

These components correspond to the northeast side of the triangle $\triangle_{1}^{p}$. Then [14] we have

$$
\operatorname{dim} V \geq \operatorname{dim} \mathcal{H}^{p-1} /(m+1) .
$$

(This is because the condition guarantees that the partial derivatives of the components of $f$ span $\mathcal{H}^{p-1}$.)

The main result of DoCarmo-Wallach $[3,17]$ asserts that $\mathcal{F}_{m}^{p}$ is nontrivial iff $m \geq 3$ and $p \geq 4$.

The following result is proved in [14] and gives the positive resolution of the so-called DoCarmo-Wallach conjecture:

Theorem 3. For $m \geq 3$ and $p \geq 4$,

$$
V^{(2,2,0, \ldots, 0)}, \ldots, V^{(2 p-2,2,0, \ldots, 0)}
$$


are not components of $\mathcal{F}^{p}$ so that we have

$$
\mathcal{F}^{p} \otimes \mathbf{C}=\sum_{(u, v) \in \triangle_{2}^{p} ; u, v \text { even }} V^{(u, v, 0, \ldots, 0)},
$$

where $\triangle_{2}^{p}$ is the closed convex triangle in $\mathbf{R}^{2}$ with vertices $(4,4),(p, p)$ and $(2 p-$ $4,4)$.

Remark. In the lowest nonrigid range $m=3$ and $p=4$, Theorem 3 has been proved by Muto [9].

\subsection{Isotropic minimal immersions}

Let $f: S^{m} \rightarrow S_{V}$ be a minimal immersion with homothety $\lambda_{p} / m$. We denote by $\beta_{k}(f)$ and $\mathcal{O}_{f}^{k}, k \leq p$, the $k$-th fundamental form and the $k$-th osculating bundle of $f$. We say that $f$ is isotropic of order $k, 2 \leq k \leq p$, if, for $2 \leq l \leq k$, we have

$$
\begin{aligned}
& \left\langle\beta_{l}(f)\left(X_{1}, \ldots, X_{l}\right), \beta_{l}(f)\left(X_{l+1}, \ldots, X_{2 l}\right)\right\rangle \\
& \quad=\left\langle\beta_{l}\left(f_{p}\right)\left(X_{1}, \ldots, X_{l}\right), \beta_{l}\left(f_{p}\right)\left(X_{l+1}, \ldots, X_{2 l}\right)\right\rangle
\end{aligned}
$$

where $X_{1}, \ldots, X_{2 l}$ are vector fields on $S^{m}$ [5]. In this case, for $2 \leq l \leq k$, the osculating bundles $\mathcal{O}_{f}^{l}$ and $\mathcal{O}_{f_{p}}^{l}$ are isomorphic with a fibrewise isometry. Restricting to the base point $o=(1,0, \ldots, 0) \in S^{m}$, as $S O(m)$-modules, we thus have

$$
\mathcal{O}_{f ; o}^{l}=\mathcal{O}_{f_{p} ; o}^{l}=\mathcal{H}_{m-1}^{l}, \quad 2 \leq l \leq k \text {. }
$$

In particular, since the osculating bundles are in the normal bundle, we obtain that, for a full minimal immersion $f: S^{m} \rightarrow S_{V}$ with homothety $\lambda_{p} / m$, isotropy of order $k$ implies that

$$
\operatorname{dim} V \geq \sum_{l=0}^{k} \operatorname{dim} \mathcal{H}_{m-1}^{l}=\operatorname{dim} \mathcal{H}_{m}^{k}
$$

(The last equality is because of branching over $S O(m) \subset S O(m+1)$.)

According to a result of [5], the space of congruence classes of full minimal immersions $f: S^{m} \rightarrow S_{V}$ with homothety $\lambda_{p} / m$ that are isotropic of order $k$, $2 \leq k \leq p$, is parametrized by a linear slice $\mathcal{M}^{p ; k}$ of $\mathcal{M}^{p}$ whose linear span $\mathcal{F}^{p ; k}$ is an $S O(m+1)$-submodule of $S^{2}\left(\mathcal{H}^{p}\right)$. We have [5]

$$
\mathcal{F}^{p ; k} \otimes \mathbf{C} \supset \sum_{(u, v) \in \triangle_{k}^{p} ; u, v \text { even }} V^{(u, v, 0, \ldots, 0)},
$$

where $\triangle_{k}^{p} \subset \triangle_{2}^{p}$ is the subtriangle with vertices $(2(k+1), 2(k+1)),(p, p)$ and $(2(p-k-1), 2(k+1))$. Moreover (and this is more difficult), for $m \geq 4$, equality holds.

Combining this with the above, we see that given a full minimal immersion $f: S^{m} \rightarrow S_{V}$ with homothety $\lambda_{p} / m$, if $\langle f\rangle \in V^{(2 l, 2 k)}, l=k, \ldots, p-k$, then ( $f$ is isotropic of order $k-1) \operatorname{dim} V \geq \operatorname{dim} \mathcal{H}_{m}^{k-1}$. 


\subsection{Representations of $S U(2)=S^{3}$}

From now on we specialize the source dimension to $m=3$. The case of the 3 sphere $S^{3}$ as a source deserves a special attention since it is itself a Lie group; the Lie group of quaternions of unit length. We write an element of $S^{3}$ as $a+b j$, where $|a|^{2}+|b|^{2}=1, a, b \in \mathbf{C}$. Associating to $a+b j \in S^{3}$ the special unitary matrix

$$
\left[\begin{array}{cc}
a & b \\
-\bar{b} & \bar{a}
\end{array}\right] \in S U(2)
$$

gives an isomorphism $S^{3} \cong S U(2)$. Viewing a complex 2-vector $(z, w) \in \mathbf{C}^{2}$ as a real 4 -vector $(x, u, y, v) \in \mathbf{R}^{4}, z=x+i y, w=u+i v$, gives an embedding of $S U(2)$ into $S O(4)$ as a normal subgroup. The orthogonal transformation $\gamma=$ $\operatorname{diag}(1,1,1,-1) \in O(4)$ (or equivalently, $\gamma: z \mapsto z, w \mapsto \bar{w})$ conjugates $S U(2)$ to the subgroup

$$
S U(2)^{\prime}=\gamma S U(2) \gamma, \quad \gamma^{-1}=\gamma
$$

of $S O(4)$ and (as simple computation shows), we have

$$
S U(2) \bigcap S U(2)^{\prime}=\{ \pm I\}
$$

and (for reasons of dimension)

$$
S U(2) \cdot S U(2)^{\prime}=S O(4)
$$

The complex irreducible $S U(2)$-modules are parametrized by the dimension of the module. More concretely, let $W_{p}, p \geq 0$, be the linear space of complex homogeneous polynomials of degree $p$ in $z$ and $w$. The standard basis in $W_{p}$ is $\left\{z^{p-q} w^{q}\right\}_{q=0}^{p} . W_{p}$ is a complex irreducible $S U(2)$-module with $\operatorname{dim} W_{p}=p+1$ and each complex irreducible $S U(2)$-module is equivalent to $W_{p}$ for some $p$. As $S U(2)$-modules, we have

$$
W_{r} \otimes W_{s}=\sum_{t=0}^{s} W_{r+s-2 t}, \quad r \geq s \geq 0
$$

Restricting from $S O(4)$ to $U(2)$, the $S O(4)$-module $\mathcal{H}^{p}$ of complex spherical harmonics on $S^{3}$ of order $p$ splits as

$$
\left.\mathcal{H}^{p}\right|_{U(2)}=\sum_{a+b=p ; a, b \geq 0} \mathcal{H}^{a, b}
$$

where $\mathcal{H}^{a, b}$ is the complex irreducible $U(2)$-module of harmonic polynomials of degree $a$ in $z, w$ and degree $b$ in $\bar{z}, \bar{w}$. (This is easily seen by writing a harmonic $p$ homogeneous polynomial in terms of the variables $z, \bar{z}, w, \bar{w}$.) The center $\Gamma \subset U(2)$ 
acts on each $\mathcal{H}^{a, b}$ as a character. Restricting further to $S U(2) \subset U(2)$, we thus obtain

$$
\left.\mathcal{H}^{p}\right|_{S U(2)}=(p+1) W_{p}
$$

as complex $S U(2)$-modules. More generally, by the local product structure (8), the $S O(4)$-module $\mathcal{H}^{p}$ splits into the tensor product

$$
\mathcal{H}^{p}=W_{p} \otimes W_{p}^{\prime}
$$

Here we use the following notation. If $W$ is an $S U(2)$-module then $W^{\prime}$ denotes the $S U(2)^{\prime}$-module obtained from $W$ by conjugating first $S U(2)^{\prime}$ back to $S U(2)$ within $S O(4)$. Moreover, if $-I$ acts on $W^{\prime}$ trivially then $W^{\prime}$ is also considered as an $S O(4)$-module with trivial action of $S U(2)$ on $W^{\prime}$. Similarly, if $-I$ acts on $W$ trivially then $W$ is also an $S O(4)$-module with $\left.W\right|_{S U(2)}$, being trivial.

In the next lemma recall that we write $V^{(u, v)}, v>0$, for $V_{4}^{(u, v)} \oplus V_{4}^{(u,-v)}$.

Lemma. Let $u \geq v \geq 1$ and $u+v$ even. Then

$$
V^{(u, v)}=W_{u-v} \otimes W_{u+v}^{\prime} \oplus W_{u+v} \otimes W_{u-v}^{\prime}
$$

and hence

$$
\left.V^{(u, v)}\right|_{S U(2)}=(u+v+1) W_{u-v} \oplus(u-v+1) W_{u+v} .
$$

Proof. The northern vertex $(u, v)$ in $\triangle_{0}^{u, v}$ is missed by the subtriangles $\triangle_{0}^{u-1, v-1}$ and $\triangle_{0}^{u+1, v-1}$ overlapping in $\triangle_{0}^{u, v-2}$. We thus obtain

$$
V^{(u, v)} \oplus\left(\mathcal{H}^{u-1} \otimes \mathcal{H}^{v-1}\right) \oplus\left(\mathcal{H}^{u+1} \otimes \mathcal{H}^{v-1}\right)=\left(\mathcal{H}^{u} \otimes \mathcal{H}^{v}\right) \oplus\left(\mathcal{H}^{u} \otimes \mathcal{H}^{v-2}\right) .
$$

We work out each tensor product using

$$
\begin{aligned}
\mathcal{H}^{r} \otimes \mathcal{H}^{s} & =\left(W_{r} \otimes W_{r}^{\prime}\right) \otimes\left(W_{s} \otimes W_{s}^{\prime}\right) \\
& =\left(W_{r+s} \oplus W_{r+s-2} \oplus \ldots \oplus W_{r-s}\right) \\
& \otimes\left(W_{r+s}^{\prime} \oplus W_{r+s-2}^{\prime} \oplus \ldots \oplus W_{r-s}^{\prime}\right), r \geq s
\end{aligned}
$$

and arrive at the stated equality.

Corollary. Let $u \geq v \geq 1$ and $u+v$ even. Then $\left.V^{(u, v)}\right|_{S U(2)}$ contains the trivial $S U(2)$-module iff $u=v$. The multiplicity of the trivial $S U(2)$-module in $V^{(u, u)}$ is $2 u+1$.

With the notation introduced above, we have

$$
V^{(u, u)}=W_{2 u} \oplus W_{2 u}^{\prime}
$$


as (complex) $S O(4)$-modules.

Finally, we make some comments on real irreducible $S U(2)$-modules. For $p$ even, $W_{p}$ is the complexification of an irreducible real $S U(2)$-submodule $R_{p}$. In fact, $R_{p}$ is the real subspace of the complex antilinear map of $W_{p}$ that sends $z^{q} w^{p-q}$ to $(-1)^{q} z^{p-q} w^{q}, q=0, \ldots, p$. The standard basis of $R_{p}$ is given by

$$
z^{p}+w^{p}, i\left(z^{p}-w^{p}\right), z^{p-1} w-z w^{p-1}, i\left(z^{p-1} w+z w^{p-1}\right), \ldots, i^{p / 2} z^{p / 2} w^{p / 2} .
$$

Using (11) and (13), we obtain the following real $S O(4)$ modules for $p$ even

$$
\mathcal{H}^{p}=R_{p} \otimes R_{p}^{\prime} \quad,\left.\quad \mathcal{H}^{p}\right|_{S U(2)}=(p+1) R_{p} \quad, \quad V^{(u, u)}=R_{2 u} \oplus R_{2 u}^{\prime} .
$$

For $p$ odd, $W_{p}$, considered as a real $S U(2)$-module, is irreducible. We denote this real representation by $\left[W_{p}\right]_{\mathbf{R}}$. Hence we obtain the following real $S O(4)$-modules for $p$ odd

$$
\mathcal{H}^{p}=\left[W_{p} \otimes W_{p}^{\prime}\right]_{\mathbf{R}} \quad,\left.\quad \mathcal{H}^{p}\right|_{S U(2)}=\frac{p+1}{2}\left[W_{p}\right]_{\mathbf{R}} \quad, \quad V^{(u, u)}=R_{2 u} \oplus R_{2 u}^{\prime} .
$$

\section{2. $S U(2)$ equivariant eigenmaps and minimal immersions}

Since $S^{3}$ acts on itself by left quaternionic multiplication, a $p$-eigenmap $f: S^{3} \rightarrow$ $S_{V}$ is $S U(2)$ equivariant if there exists a homomorphism $\rho_{f}: S U(2) \rightarrow S O(V)$ such that

$$
f \circ L_{g}=\rho_{f}(g) \circ f, \quad g \in S U(2),
$$

where $L_{g}$ is left quaternionic multiplication on $S^{3}$ by $g$ as a quaternion. Clearly, a $p$-eigenmap $f$ is $S U(2)$ equivariant iff $f \circ \gamma$ is $S U(2)^{\prime}$ equivariant. The spaces of congruence classes of full $S U(2)$ (resp. $\left.S U(2)^{\prime}\right)$ equivariant $p$-eigenmaps are parametrized by the linear slices

$$
\left(\mathcal{L}^{p}\right)^{S U(2)}=\mathcal{L}^{p} \cap\left(\mathcal{E}^{p}\right)^{S U(2)} \quad\left(\operatorname{resp} .\left(\mathcal{L}^{p}\right)^{S U(2)^{\prime}}=\mathcal{L}^{p} \cap\left(\mathcal{E}^{p}\right)^{S U(2)^{\prime}}\right) .
$$

We can find $\left(\mathcal{E}^{p}\right)^{S U(2)}$ from the decomposition formula (3) by setting $m=3$, restricting both sides to $S U(2)$ and counting the trivial components. In fact, according to (14):

$$
\left(V^{(u, u)}\right)^{S U(2)}=R_{2 u}^{\prime} \otimes \mathbf{C}
$$

as $S U(2)^{\prime}$-modules. In view of this, (3) and Corollary in 1.5, we have

$$
\begin{aligned}
\left(\mathcal{E}^{p} \otimes \mathbf{C}\right)^{S U(2)} & =\sum_{k=1}^{[p / 2]}\left(V^{(2 k, 2 k)}\right)^{S U(2)} \\
& =\sum_{k=1}^{[p / 2]}\left(R_{4 k}^{\prime} \otimes \mathbf{C}\right)
\end{aligned}
$$


so that, as real $S U(2)^{\prime}$-modules:

$$
\left(\mathcal{E}^{p}\right)^{S U(2)}=\sum_{k=1}^{[p / 2]} R_{4 k}^{\prime} .
$$

Similarly

$$
\left(\mathcal{E}^{p}\right)^{S U(2)^{\prime}}=\sum_{k=1}^{[p / 2]} R_{4 k}
$$

as real $S U(2)$-modules. In the lowest nonrigid range $p=2$ (for eigenmaps), $\triangle_{1}^{p}$ in (3) reduces to the single point $(2,2)$ so that we have

$$
\mathcal{E}^{2}=\left(\mathcal{E}^{2}\right)^{S U(2)} \oplus\left(\mathcal{E}^{2}\right)^{S U(2)^{\prime}}=R_{4}^{\prime} \oplus R_{4}
$$

as $S O(4)$-modules. Thus the moduli space $\mathcal{L}^{2}$ is 'split' by two 5 -dimensional orthogonal slices $\left(\mathcal{L}^{2}\right)^{S U(2)}=\mathcal{L}^{2} \cap R_{4}^{\prime}$ and $\left(\mathcal{L}^{2}\right)^{S U(2)^{\prime}}=\mathcal{L}^{2} \cap R_{4}$ parametrizing $S U(2)$ and $S U(2)^{\prime}$ equivariant quadratic eigenmaps. It is now a crucial observation to be generalized below that $\mathcal{L}^{2}$ is the convex hull of these slices [13].

Remark. As a convex set $\left(\mathcal{L}^{2}\right)^{S U(2)} \cong\left(\mathcal{L}^{2}\right)^{S U(2)^{\prime}}$ is the convex hull of a projective plane embedded into $S^{4}$ as the Veronese surface [14]. More generally, for $p$ even,

$$
\left(\mathcal{L}^{p}\right)^{S U(2)}=\left\{C_{0} \in S^{2}\left(R_{p}\right) \mid \operatorname{trace} C_{0}=0 \text { and } C_{0}+I \geq 0\right\} .
$$

This follows from $\left.\mathcal{H}^{p}\right|_{S U(2)}=(p+1) R_{p}$ (cf. (14)) and Schur's lemma applied to symmetric endomorphisms of $\mathcal{H}^{p}$ in $\left(\mathcal{E}^{p}\right)^{S U(2)}$ that commute with the action of $S U(2)$.

The situation is analogous for minimal immersions. We have

$$
\left(\mathcal{M}^{p}\right)^{S U(2)}=\mathcal{M}^{p} \cap\left(\mathcal{F}^{p}\right)^{S U(2)},
$$

and similarly for $S U(2)^{\prime}$. Moreover, as $S U(2)^{\prime}$-modules

$$
\begin{aligned}
\left(\mathcal{F}^{p} \otimes \mathbf{C}\right)^{S U(2)} & =\sum_{k=2}^{[p / 2]}\left(V^{(2 k, 2 k)}\right)^{S U(2)} \\
& =\sum_{k=2}^{[p / 2]}\left(R_{4 k}^{\prime} \otimes \mathbf{C}\right) .
\end{aligned}
$$

As for eigenmaps, we see that the $S U(2)$ and $S U(2)^{\prime}$ equivariant minimal immersions correspond to the northwest side of $\triangle_{2}^{p}$ in (5). As real $S U(2)^{\prime}$-modules

$$
\left(\mathcal{F}^{p}\right)^{S U(2)}=\sum_{k=2}^{[p / 2]} R_{4 k}^{\prime} .
$$


In particular, counting dimensions

$$
\begin{aligned}
\operatorname{dim}\left(\mathcal{M}^{p}\right)^{S U(2)} & =\operatorname{dim}\left(\mathcal{F}^{p}\right)^{S U(2)} \\
& =\sum_{k=2}^{[p / 2]}(4 k+1) \\
& =(2[p / 2]+5)([p / 2]-1) .
\end{aligned}
$$

This formula has been derived in [2] using a 'heuristic argument'.

In the lowest nonrigid range $p=4$ (for minimal immersions):

$$
\mathcal{F}^{4}=\left(\mathcal{F}^{4}\right)^{S U(2)} \oplus\left(\mathcal{F}^{4}\right)^{S U(2)^{\prime}}=R_{8}^{\prime} \oplus R_{8}
$$

as real $S O(4)$-modules. Intersecting with the moduli space we see that $\mathcal{M}^{4}$ is 'split' by two 9-dimensional orthogonal slices $\left(\mathcal{M}^{4}\right)^{S U(2)}=\mathcal{M}^{4} \cap R_{8}^{\prime}$ and $\left(\mathcal{M}^{4}\right)^{S U(2)^{\prime}}=$ $\mathcal{M}^{4} \cap R_{8}$ corresponding to $S U(2)$ and $S U(2)^{\prime}$ equivariant minimal immersions. It is also clear that precomposing quartic minimal immersions with $\gamma$ has the effect of interchanging $R_{8}$ and $R_{8}^{\prime}$.

Let $\mathcal{B}_{1}^{p}=\left(\partial \mathcal{M}^{p}\right)^{S U(2)}$ and $\mathcal{B}_{2}^{p}=\left(\partial \mathcal{M}^{p}\right)^{S U}(2)^{\prime}$. Thus $\mathcal{B}_{1}^{p}$ (resp. $\left.\mathcal{B}_{2}^{p}\right)$ parametrize the boundary type $S U(2)$ (resp. $\left.S U(2)^{\prime}\right)$ equivariant minimal immersions $f: S^{3} \rightarrow$ $S_{V}$ with homothety $\lambda_{p} / 3$.

Theorem 4. For $p \geq 4, \partial \mathcal{M}^{p} \cap V^{(2 k, 2 k)}$ is the union of segments with one endpoint on $\mathcal{B}_{1}^{p} \cap V^{(2 k, 2 k)}$ and the other on $\mathcal{B}_{2}^{p} \cap V^{(2 k, 2 k)}$. Equivalently, every full (boundary) minimal immersion $f: S^{3} \rightarrow S_{V}$ of degree $p$ such that $\langle f\rangle \in V^{(2 k, 2 k)}$ is congruent to one of the form $\left(\sqrt{\lambda_{1}} f_{1}, \sqrt{\lambda_{2}} f_{2} \circ \gamma\right): S^{3} \rightarrow S_{V_{1} \times V_{2}}, \lambda_{1}+\lambda_{2}=1$, $\lambda_{1}, \lambda_{2} \geq 0$, where $f_{1}: S^{3} \rightarrow S_{V_{1}}$ and $f_{2}: S^{3} \rightarrow S_{V_{2}}$ are full $S U(2)$ equivariant (boundary) minimal immersions with $\left\langle f_{1}\right\rangle,\left\langle f_{2}\right\rangle \in R_{4 k}^{\prime} \subset V^{(2 k, 2 k)}$.

Proof. The connecting lemma establishes the equivalence of the two statements. To prove the first it is enough to show that any segment connecting $\mathcal{B}_{1}^{p} \cap V^{(2 k, 2 k)}$ and $\mathcal{B}_{2}^{p} \cap V^{(2 k, 2 k)}$ is entirely contained in the boundary of $\mathcal{M}^{p}$. Let $f_{1}$ and $f_{2}$ as in the second statement and let $\langle f\rangle$ be in the interior of the segment connecting $\left\langle f_{1}\right\rangle$ and $\left\langle f_{2} \circ \gamma\right\rangle$. We need to show that $\langle f\rangle \in \partial \mathcal{M}^{p}$. By the connecting lemma cited above, we have

$$
V_{f}=V_{f_{1}}+V_{f_{2} \circ \gamma} .
$$

Since $f_{1}$ and $f_{2}$ are $S U(2)$ equivariant and of boundary type, $V_{f_{1}}$ is a proper $S U(2)$-submodule of $\mathcal{H}^{p}$ and $V_{f_{2} \circ \gamma}$ is a proper $S U(2)^{\prime}$-submodule of $\mathcal{H}^{p}=W_{p} \otimes W_{p}^{\prime}$ (for simplicity we complexify again). We need the following elementary statement whose proof is an easy application of Schur's lemma.

Lemma. Let $G$ be a compact Lie group, $R$ an irreducible $G$-module and $W$ a trivial $G$-module. Then any $G$-submodule $Z$ of $R \otimes W$ is of the form $Z=R \otimes W_{0}$, where $W_{0} \subset W$ is a linear subspace. 
Returning to our previous setting, we have

$$
V_{f_{1}}=W_{p} \otimes W_{0}^{\prime} \quad \text { and } \quad V_{f_{2} \circ \gamma}=W_{0} \otimes W_{p}^{\prime}
$$

where $W_{0} \subset W_{p}$ and $W_{0}^{\prime} \subset W_{p}^{\prime}$ are proper linear subspaces. Hence

$$
V_{f}=V_{f_{1}}+V_{f_{2} \circ \gamma}=W_{p} \otimes W_{0}^{\prime}+W_{0} \otimes W_{p}^{\prime} .
$$

But $W_{0} \otimes W_{0}^{\prime}$ is equal to the overlap of both subspaces and hence $V_{f}$ can never have the full dimension. Thus $f$ is of boundary type.

Theorem 4 gives a complete description of full minimal immersions $f: S^{3} \rightarrow$ $S_{V}$ with homothety $\lambda_{p} / 3$ whose parameter point $\langle f\rangle$ is in one of the irreducible components $V^{(4,4)}, V^{(6,6)}, \ldots, V^{(2[p / 2], 2[p / 2])}$ corresponding to the northwest side of the triangle $\triangle_{2}^{p}$ in (5). It states that such minimal immersions are obtained from $S U(2)$ equivariant ones by the prescription given in the connecting lemma. In particular, in the lowest nonrigid range $p=4, \triangle_{2}^{4}$ collapses to the single point $(4,4)$ so that Theorem 4 completely describes all full quartic minimal immersions in terms of $S U(2)$ equivariant ones. The proof of Theorem 4 also gives all possible range dimensions of such minimal immersions for $p \geq 4$. This gives a partial answer to a problem posed by DoCarmo: What are the possible (in particular, minimum) range dimensions of all spherical minimal immersions?

Theorem 5. Let $f: S^{3} \rightarrow S_{V}$ be a full minimal immersion of degree $p \geq 4$ and assume that $\langle f\rangle \in V^{(2 k, 2 k)}$. Then the possible range dimensions of $f$ (plus one) are

a) For $p$ even

$\operatorname{dim} V=(p+1)^{2}-r s, \quad$ with $1 \leq r, s \leq p-[(p+1) / 4]$ or

$\operatorname{dim} V=(p+1) r, \quad$ with $\quad[(p+1) / 4]+1 \leq r \leq p+1$ (for the equivariant ones).

b) For $p$ odd

$\operatorname{dim} V=(p+1)^{2}-r s, \quad$ with $\quad 1 \leq r, s \leq p-[(p+1) / 8]$ or

$\operatorname{dim} V=(p+1) r$, with $[(p+1) / 8]+1 \leq r \leq p+1$ (for the equivariant ones).

Proof. It follows from the proof of Theorem 4 that $\operatorname{dim} V_{f}=(p+1) \operatorname{dim} W_{0}^{\prime}+$ $\operatorname{dim} W_{0}(p+1)-\operatorname{dim} W_{0}^{\prime} \operatorname{dim} W_{0}$. Furthermore, notice that it follows from (15) that for $p$ odd, the dimension of $W_{0}$ and $W_{0}^{\prime}$ must be even. But there are further restrictions on the possible dimensions of equivariant embeddings. In [15] it was proved that if $f: S^{3} \rightarrow S_{V}$ is a full $S U(2)$ equivariant minimal immersion of degree $p$ and $\langle f\rangle \in V^{(2 k, 2 k)}$ then $\operatorname{dim} V \geq(p+1)^{2} / 4$. Of course for the equivariant ones we also need that $\operatorname{dim} V$ is divisible by $p+1$ if $p$ is even and by $2(p+1)$ if $p$ is odd. This easily implies the claim in the theorem. 
Remark. For $p=4$ and $p=5,\left(\mathcal{F}^{p}\right)^{S U(2)}$ consists only of a single irreducible summand. But starting with $p=6$, it has at least two irreducible summands, in particular $\left(\mathcal{F}^{6}\right)^{S U}(2)=R_{8}^{\prime} \oplus R_{12}^{\prime}$. In [15] it was shown that if $p=6$ and if $\langle f\rangle$ lies in $R_{8}^{\prime}$ or in $R_{12}^{\prime}$, then $\operatorname{dim} V \geq 14$. The connecting lemma hence shows that any point in the convex hull of $\mathcal{M}^{6} \cap R_{8}^{\prime}$ with $\mathcal{M}^{6} \cap R_{12}^{\prime}$ also has $\operatorname{dim} V \geq 14$. But in [2] an example was constructed of an equivariant minimal isometric immersion with $p=6$ and $\operatorname{dim} V=7$ (and was shown to be unique among such equivariant isometric immersions). It follows that it cannot be in the convex hull of $\mathcal{M}^{6} \cap R_{8}^{\prime}$ with $\mathcal{M}^{6} \cap R_{12}^{\prime}$, i.e this immersion must "bulge out" in the moduli space. Thus the moduli space for $p \geq 6$ must have a much more complicated structure than for $p=4$. It was conjectured in [2] that this degree 6 immersion into $S^{6}$ is the only degree 6 immersion with $\operatorname{dim} V=7$. The above remarks show that a proof of this fact must be much more complicated than the proof of theorem B.

\section{Quartic minimal immersions}

From now on we let $p=4$ (and $m=3$ ). Theorem 4 asserts that $\mathcal{M}^{4}$ is the convex hull of $\left(\mathcal{M}^{4}\right)^{S U(2)}$ and $\left(\mathcal{M}^{4}\right)^{S U(2)^{\prime}}$ corresponding to $S U(2)$ and $S U(2)^{\prime}$ equivariant quartic minimal immersions. The possible range dimensions for a full $S U(2)$-equivariant quartic minimal immersion $f: S^{3} \rightarrow S_{V}$ are

$$
\operatorname{dim} V=5,10,15,20,25 .
$$

This is because $V$ is an $S U(2)$-submodule of $\left.\mathcal{H}^{4}\right|_{S U(2)}=5 R_{4}$ so that it must be a multiple of $R_{4}$. The range dimension 5 is not realized. This follows from a general theorem of Moore [8] or by easy computation in the use of the equivariant construction below. Thus Theorem 5 gives all possible range dimensions of full quartic minimal immersions $f: S^{3} \rightarrow S_{V}$ :

$$
\operatorname{dim} V=10,15,16,19,20,21,22,23,24,25 .
$$

We will see later that the range dimensions 10,15 , and 20 actually occur for full $S U(2)$ equivariant quartic minimal immersions so that, without equivariance, all the rest of the range dimensions above are realized. Notice that it also follows from the proof of Theorem 5 that $\operatorname{dim} V=10,15$ and 20 can only occur if the minimal immersion is $S U(2)$ equivariant or $S U(2)^{\prime}$ equivariant. In [2] it was shown that there exists a unique $S U(2)$ equivariant minimal immersion with $\operatorname{dim} V=10$ (up to precomposition with an isometry of the domain) and hence Theorems A and B in the introduction follow.

We say that an equivariant minimal immersion is of type I, II, or III if the ambient dimension is $\operatorname{dim} V=10,15$, or 20 . We denote by I, II, and III the set of all minimal immersions of type I, II, and III. Similarly for $\mathbf{I}^{\prime}, \mathbf{I I}^{\prime}$, and $\mathbf{I I I}^{\prime}$ in $\partial\left(\mathcal{M}^{4}\right)^{S U(2)^{\prime}}$. It follows also, that for example the immersions with $\operatorname{dim} V=16$ in $\partial \mathcal{M}^{4}$ can only be obtained by connecting an immersion in $\mathbf{I}$ with one in $\mathbf{I}^{\prime}$. 
Furthermore, if we connect a linear slice $\mathcal{M}_{f}$ in III with another linear slice $\mathcal{M}_{f^{\prime}}$ in $\mathbf{I I I}^{\prime}$, we obtain a 13 dimensional linear slice in $\partial \mathcal{M}^{4}$, which is the largest dimensional linear slice in the boundary of $\mathcal{M}^{4}$. The equivariant immersions must have an embedded image since they are given by orbits under the action of $S U(2)$ and $S U(2)^{\prime}$, in fact the orbit of a polynomial $p \in \mathcal{H}^{4}$ is diffeomorphic to $S U(2) / G$ where $G$ is the isotropy group of $p$. From [2] it follows that the possible isotropy groups are $D_{2}^{*}, Z_{4}$ and $Z_{2}, Z_{2}$ being the principal isotropy group. Hence in the equivariant case the images are embedded $S^{3} / D_{2}^{*}$, lens spaces $S^{3} / Z_{4}$, or projective spaces $\mathbf{R} P^{3}$. We will see that all three cases actually occur.

Since $\left(\mathcal{M}^{4}\right)^{S U(2)^{\prime}}$ is the $\gamma$-copy of $\left(\mathcal{M}^{4}\right)^{S U(2)}$, it remains to describe the latter and this is our main purpose in this section. For simplicity we denote $\mathcal{B}=$ $\left(\mathcal{M}^{4}\right)^{S U(2)}$ and from now on all minimal immersions will be $S U(2)$ equivariant. The $S O(4)$ action on $\left(\mathcal{M}^{4}\right)^{S U(2)}$ reduces to an action of $S U(2)^{\prime}$ and hence $\mathbf{I}$, II, and III are each union of $S U(2)^{\prime}$ orbits. The action of $S U(2)^{\prime}$ on $R_{8}^{\prime}=\left(\mathcal{F}^{4}\right)^{S U(2)}$ is the action on polynomials in $z, w$ of degree 8 . The orbits are all 3-dimensional, except one orbit, the one through $z^{4} w^{4}$, which gives rise to a minimal isometric embedding of $\mathbf{R} P^{2}$ into a sphere in $R_{8}^{\prime}$. It follows from [2] that the possible finite isotropy groups of $S U(2)^{\prime}$ acting on $R_{8}^{\prime}$ and hence also on $\partial\left(\mathcal{M}^{4}\right)^{S U(2)}$ consist of the cyclic group $Z_{8}$ (the principal isotropy group), the binary dihedral groups $D_{2}^{*}$, $D_{3}^{*}$ and $D_{4}^{*}$, and the binary octahedral group $O^{*}$. As was observed in [2], many of the orbits are again minimal isometric immersions in their respective spheres.

We now turn to the 'equivariant construction' for $S U(2)$ that provides an explicit description of all $S U(2)$ equivariant minimal immersions as (constant curvature) $S U(2)$ orbits of polynomials in $S U(2)$-submodules of $\mathcal{H}^{p}$. We first summarize some of the results in [2]. Each equivariant construction used here is based on an $S U(2)$-submodule of $\mathcal{H}^{p}$, where we now (briefly) return to the general case $p \geq 4$. As a first example we take this to be $W_{p}$. Consider a polynomial $\xi \in W_{p}$, of unit length. In terms of the standard basis in $W_{p}$, we write

$$
\xi(z, w)=c_{0} z^{p}+c_{1} z^{p-1} w+\ldots+c_{p} w^{p}, \quad c_{0}, \ldots, c_{p} \in \mathbf{C} .
$$

Let $f_{\xi}: S^{3} \rightarrow S_{W_{p}}$ be the orbit map:

$$
f_{\xi}(g)=g \cdot \xi=\xi \circ L_{g^{-1}}, \quad g \in S U(2)=S^{3} .
$$

More explicitly, setting $g=a+b j \in S^{3}$, we have

$$
f_{\xi}(a+b j)(z, w)=\xi(\bar{a} z-b w, \bar{b} z+a w), \quad z, w \in \mathbf{C} .
$$

It is important to note here that $f_{\xi}$ is equivariant with respect to the conjugate subgroup $S U(2)^{\prime}$ (and not with respect to $S U(2)$ ). More precisely, by the identifications we made, we have

$$
f_{\xi}\left(\gamma\left[\begin{array}{cc}
\alpha & -\beta \\
\bar{\beta} & \bar{\alpha}
\end{array}\right] \gamma \cdot(a+b j)\right)=\left[\begin{array}{cc}
\alpha & \beta \\
-\bar{\beta} & \bar{\alpha}
\end{array}\right] \cdot f_{\xi}(a+b j) .
$$


Note that the matrices differ by conjugation with diag $(1,1,-1,-1)$ (that preserves the local product structure (8)). For this reason, we will usually make $f_{\xi} S U(2)$ equivariant by precomposing it with $\gamma$.

$f_{\xi}$ is minimal iff

$$
\begin{aligned}
& \sum_{q=0}^{p}(2 q-p)^{2}(p-q) ! q !\left|c_{q}\right|^{2}=\frac{p(p+2)}{3}, \\
& \sum_{q=0}^{p}(p-q) ! q !\left|c_{q}\right|^{2}=1, \\
& \sum_{q=0}^{p-2}(q+2) !(p-q) ! c_{q} \bar{c}_{q+2}=0, \\
& \sum_{q=0}^{p-1}(p-2 q-1)(q+1) !(p-q) ! c_{q} \bar{c}_{q+1}=0 .
\end{aligned}
$$

The first two equations are real and the last two are complex so that we have 6 constraints on the $2(p+1)$ real variables $\Re\left(c_{q}\right)$ and $\Im\left(c_{q}\right), q=0, \ldots, p$.

For $p$ even, we can take $R_{p}$ instead of $W_{p}$ by requiring $\xi \in R_{p}$ (so that $f_{\xi}$ will actually map into $S_{R_{p}}$ ). Using the standard basis in $R_{p}$, we obtain that this additional requirement translates into

$$
c_{p}=\bar{c}_{0}, \quad c_{p-1}=-\bar{c}_{1}, \ldots, c_{(p / 2)+1}=(-1)^{(p / 2)+1} \bar{c}_{(p / 2)-1}, \quad c_{p / 2}=i^{p / 2} t, t \in \mathbf{R} .
$$

We incorporate these $p+1$ additional constraints by writing

$$
\xi(z, w)=c_{0} z^{p}+\bar{c}_{0} w^{p}+c_{1} z^{p-1} w-\bar{c}_{1} z w^{p-1}+\ldots+i^{p / 2} t z^{p / 2} w^{p / 2} .
$$

The system of equations for minimality of $f_{\xi}$ thus reduces to

$$
\begin{aligned}
& \sum_{q=0}^{(p / 2)-1} 2(2 q-p)^{2}(p-q) ! q !\left|c_{q}\right|^{2}=\frac{p(p+2)}{3}, \\
& \sum_{q=0}^{(p / 2)-1} 2(p-q) ! q !\left|c_{q}\right|^{2}+((p / 2) !)^{2} t^{2}=1, \\
& (p / 2)-3 \\
& \sum_{q=0}^{(p / 2)} 2(q+2) !(p-q) ! c_{q} \bar{c}_{q+2}+(-1)^{(p / 2)+1}(((p / 2)+1) !)^{2} c_{(p / 2)-1}^{2} \\
& +(-i)^{p / 2} 2(p / 2) !((p / 2)+2) ! c_{(p / 2)-2} t=0, \\
& \sum_{q=0}^{(p / 2)-2}(p-2 q-1)(q+1) !(p-q) ! c_{q} \bar{c}_{q+1}+(-i)^{p / 2}(p / 2) !((p / 2)+1) ! c_{(p / 2)-1} t=0 .
\end{aligned}
$$


As a first explicit example we now show that there is no $S U(2)$ equivariant quartic minimal immersion with range $V=R_{4}$. Indeed any such should come from the equivariant construction above for $p=4$. The equations for minimality reduce to

$$
\begin{aligned}
96\left|c_{0}\right|^{2}+6\left|c_{1}\right|^{2} & =1 \\
48\left|c_{0}\right|^{2}+12\left|c_{1}\right|^{2}+4 t^{2} & =1 \\
3 c_{1}^{2}+8 c_{0} t & =0 \\
6 c_{0} \bar{c}_{1}-c_{1} t & =0 .
\end{aligned}
$$

These equations are inconsistent and we are done.

Next we turn to the description of type I minimal immersions. These are obtained from the equivariant construction applied to $W_{4}=2 R_{4}$. Setting $p=4$ the constraints for $W_{4}$ specialize to

$$
\begin{aligned}
& 48\left|c_{0}\right|^{2}+3\left|c_{1}\right|^{2}+3\left|c_{3}\right|^{2}+48\left|c_{4}\right|^{2}=1, \\
& 24\left|c_{0}\right|^{2}+6\left|c_{1}\right|^{2}+4\left|c_{2}\right|^{2}+6\left|c_{3}\right|^{2}+24\left|c_{4}\right|^{2}=1, \\
& 4 c_{0} \bar{c}_{2}+3 c_{1} \bar{c}_{3}+4 c_{2} \bar{c}_{4}=0, \\
& 6 c_{0} \bar{c}_{1}+c_{1} \bar{c}_{2}-c_{2} \bar{c}_{3}-6 c_{3} \bar{c}_{4}=0 .
\end{aligned}
$$

This system has solutions, for example

$$
c_{0}=\sqrt{6} / 24, c_{1}=0, c_{2}=\sqrt{2} / 4, c_{3}=0, c_{4}=-\sqrt{6} / 24 .
$$

To work out the orbit map $f_{\xi}: S^{3} \rightarrow S_{W_{4}}$, we identify $W_{4}$ with $\mathbf{C}^{5}$ by the orthonormal basis

$$
z^{4} / \sqrt{24}, z^{3} w / \sqrt{6}, z^{2} w^{2} / 2, z w^{3} / \sqrt{6}, w^{4} / \sqrt{24} .
$$

We obtain (replacing the variable $a+b j$ with $z+w j$ in $S^{3}$, precomposing with $\gamma$ and up to an isometry on the range) the full quartic minimal immersion $\mathcal{I}: S^{3} \rightarrow S^{9}$ of type I, given by

$$
\begin{aligned}
\mathcal{I}(z, w) & =\left((1 / \sqrt{2})\left(z^{4}-\bar{w}^{4}\right), \sqrt{6} z^{2} \bar{w}^{2}\right. \\
& \sqrt{2}\left(z^{3} w+\bar{z} \bar{w}^{3}\right), \sqrt{6}\left(z \bar{z}^{2} w-\bar{z} w^{2} \bar{w}\right), \\
& \left.\sqrt{3 / 2}\left(z^{2} w^{2}-\bar{z}^{2} \bar{w}^{2}\right),(1 / \sqrt{2})\left(|z|^{4}-4|z|^{2}|w|^{2}+|w|^{4}\right)\right) .
\end{aligned}
$$

(The first four coordinates are complex, the fifth is purely imaginary and the sixth is real so that $\mathcal{I}$ maps into $\mathbf{C}^{4} \times(i \mathbf{R}) \times \mathbf{R}=\mathbf{R}^{10}$.) An important property of $\mathcal{I}$ is that its components are orthogonal with the same norm. 
According to a rigidity result in [2], up to isometries on the source and the range, this is the only type I minimal immersion and the image of the immersion is an embedded $S^{3} / D_{2}^{*}$. Hence

$$
\mathbf{I}=S U(2)^{\prime} \cdot\langle\mathcal{I}\rangle,
$$

where we used $S U(2)$ equivariance of $\mathcal{I}$. For the next result we recall that the octahedral manifold [2] is the quotient $S^{3} / \mathbf{O}^{*}$, where $\mathbf{O}^{*}$ is the binary octahedral group (that is the twofold cover of the group of symmetries of the regular octahedron along $\left.S^{3} \rightarrow S O(3)\right)$.

Theorem 6. I is a single $S U(2)^{\prime}$-orbit. Furthermore, this orbit is an octahedral manifold $S^{3} / \mathbf{O}^{*}$ embedded minimally in an 8-sphere of $R_{8}^{\prime}$. This embedding is given by a degree 8 equivariant immersion of $S^{3}$.

To prove the second part, we will have to show that $\mathbf{O}^{*}$ is the isotropy group of the $S U(2)^{\prime}$ action at $\langle\mathcal{I}\rangle$, a computation which we postpone for the moment. Once this is done, it follows that the orbit $S^{3} / \mathbf{O}^{*}$ must be minimally embedded in a sphere since according to [2] there exists only one orbit with isotropy $\mathbf{O}^{*}$ which is hence an isolated exceptional orbit and hence must be minimal. Or, as was first observed in [18], $S^{3} / \mathbf{O}^{*}$ is isotropy irreducible and hence for every invariant polynomial the orbit construction must give rise to an isometric embedding up to scaling.

We will first consider the type II immersions:

Theorem 7. We have

$$
\operatorname{dim} \mathbf{I I} \leq 6
$$

The set II splits into the disjoint union

$$
\mathbf{I I}=\mathbf{I I}_{0} \cup \mathbf{I I}_{1}
$$

corresponding to linearly rigid and nonrigid quartic minimal immersions. We have

$$
\operatorname{dim} \mathbf{I I}_{0}=6 .
$$

and

$$
\mathbf{I I}_{1}=S U(2)^{\prime} \cdot \mathcal{D},
$$

where $\mathcal{D}$ is a flat 2-dimensional disk with boundary circle on the octahedral manifold $\mathbf{I}$.

Proof. We show (18) by a careful dimension computation. For type II minimal immersions the $S U(2)$-module is $3 R_{4}$. Since $R_{4}$ can be thought of as the $S U(2)$ module of quartic polynomials

$$
\xi(z, w)=c_{0} z^{4}+\bar{c}_{0} w^{4}+c_{1} z^{3} w-\bar{c}_{1} z w^{3}-t z^{2} w^{2}, c_{0}, c_{1} \in \mathbf{C}, t \in \mathbf{R},
$$


we look upon a general element of $3 R_{4}$ as a triple:

$$
\left(\begin{array}{c}
a_{0} z^{4}+\bar{a}_{0} w^{4}+a_{1} z^{3} w-\bar{a}_{1} z w^{3}-r z^{2} w^{2} \\
b_{0} z^{4}+\bar{b}_{0} w^{4}+b_{1} z^{3} w-\bar{b}_{1} z w^{3}-s z^{2} w^{2} \\
c_{0} z^{4}+\bar{c}_{0} w^{4}+c_{1} z^{3} w-\bar{c}_{1} z w^{3}-t z^{2} w^{2}
\end{array}\right)
$$

where $a_{0}, a_{1}, b_{0}, b_{1}, c_{0}, c_{1} \in \mathbf{C}$ and $r, s, t \in \mathbf{R}$. The decomposition $3 R_{4}(=V)$ is not unique, in fact, $S O(3)$ acts on $3 R_{4}$ in a natural way. Thus, rotating $(r, s, t) \in \mathbf{R}^{3}$, we may assume that $r=s=0$ and $t \geq 0$. We still have the freedom to rotate along the third axis. This amounts to the change

$$
\begin{aligned}
a_{0} & \mapsto \cos \alpha \cdot a_{0}-\sin \alpha \cdot b_{0}, \\
b_{0} & \mapsto \sin \alpha \cdot a_{0}+\cos \alpha \cdot b_{0},
\end{aligned}
$$

and similarly for $a_{1}$ and $b_{1}$. The equations for minimality are

$$
\begin{aligned}
96\left(\left|a_{0}\right|^{2}+\left|b_{0}\right|^{2}+\left|c_{0}\right|^{2}\right)+6\left(\left|a_{1}\right|^{2}+\left|b_{1}\right|^{2}+\left|c_{1}\right|^{2}\right) & =1, \\
48\left(\left|a_{0}\right|^{2}+\left|b_{0}\right|^{2}+\left|c_{0}\right|^{2}\right)+12\left(\left|a_{1}\right|^{2}+\left|b_{1}\right|^{2}+\left|c_{1}\right|^{2}\right)+4 t^{2} & =1, \\
3\left(a_{1}^{2}+b_{1}^{2}+c_{1}^{2}\right)+8 c_{0} t & =0, \\
6\left(a_{0} \bar{a}_{1}+b_{0} \bar{b}_{1}+c_{0} \bar{c}_{1}\right)-c_{1} t & =0 .
\end{aligned}
$$

Note that these equations are invariant under the action (22) of $S O(2)$. For fixed $t \in \mathbf{R}$ we can solve the first two equations and obtain

$$
\begin{aligned}
& \left|a_{0}\right|^{2}+\left|b_{0}\right|^{2}+\left|c_{0}\right|^{2}=r_{0}(t)^{2} \\
& \left|a_{1}\right|^{2}+\left|b_{1}\right|^{2}+\left|c_{1}\right|^{2}=r_{1}(t)^{2}
\end{aligned}
$$

where

$$
\begin{aligned}
& r_{0}(t)^{2}=\frac{1}{144}\left(1+4 t^{2}\right) \\
& r_{1}(t)^{2}=\frac{1}{18}\left(1-8 t^{2}\right) .
\end{aligned}
$$

The second equation reduces the range of $t$ to

$$
0 \leq t \leq 1 / \sqrt{8}
$$

If $t=1 / \sqrt{8}$ then $a_{1}=b_{1}=c_{1}=0$. The third equation in (23) gives $c_{0}=0$ (the fourth is automatically satisfied) so that we have

$$
\left|a_{0}\right|^{2}+\left|b_{0}\right|^{2}=1 / 96 \text {. }
$$


We obtain that, for $t=1 / \sqrt{8}$, the solution set is the 3 -sphere (of radius $1 / \sqrt{96}$ ). The action of $S O(2)$ on $\left(a_{0}, b_{0}\right)$ (whose orbits are essentially given by the Hopf fibration) reduces this to a 2 -dimensional solution set.

Now let $0 \leq t<1 / \sqrt{8}$. Since both radii $r_{0}(t)$ and $r_{1}(t)$ are positive, the first two equations above say that

$$
\left(a_{0}, b_{0}, c_{0}\right) \in S_{r_{0}(t)}^{5} \quad \text { and } \quad\left(a_{1}, b_{1}, c_{1}\right) \in S_{r_{1}(t)}^{5}
$$

in two copies of $\mathbf{C}^{3}$. If $t=0$ then the third and fourth equations in (23) reduce to

$$
a_{1}^{2}+b_{1}^{2}+c_{1}^{2}=0
$$

and

$$
a_{0} \bar{a}_{1}+b_{0} \bar{b}_{1}+c_{0} \bar{c}_{1}=0 .
$$

The first of these is a complex quadric that intersected with $S_{1 / \sqrt{18}}^{5}$ gives a smooth 3 -dimensional manifold for $\left(a_{1}, b_{1}, c_{1}\right)$. (In fact, this is the real projective space.) For fixed $\left(a_{1}, b_{1}, c_{1}\right)$, the second equation is a complex plane that intersected with $S_{1 / 12}^{5}$ gives a great 3 -sphere. Putting these together, the product is a 6 dimensional manifold on which $S O(2)$ acts without fixed points. The quotient gives a 5 -dimensional solution set.

Finally, let $0<t<1 / \sqrt{8}$. Given $\left(a_{1}, b_{1}, c_{1}\right) \in S_{r_{1}(t)}^{5}$, we use the third equation in $(23)$ to get

$$
c_{0}=-\frac{3}{8 t}\left(a_{1}^{2}+b_{1}^{2}+c_{1}^{2}\right) .
$$

The fourth equation in (23) is an affine complex plane

$$
a_{0} \bar{a}_{1}+b_{0} \bar{b}_{1}+c_{0} \bar{c}_{1}=\frac{1}{6} c_{1} t
$$

that, intersected with $S_{r_{0}(t)}^{5}$ and knowing the value of $c_{0}$, reduces the solution set for $\left(a_{0}, b_{0}, c_{0}\right)$ to at most one dimension. This is because $a_{1}$ and $b_{1}$ cannot vanish simultaneously. (Indeed, if $a_{1}=b_{1}=0$ then $c_{0} \bar{c}_{1}=-3 c_{1}^{2} \bar{c}_{1} /(8 t)=-3\left|c_{1}\right|^{2} c_{1} /(8 t)$. On the other hand, $c_{0} \bar{c}_{1}=c_{1} t / 6$. Combining these we obtain $t=0$; a contradiction.) This, combined with the 5 -dimensional solution set for $\left(a_{1}, b_{1}, c_{1}\right)$ gives a 6 -dimensional solution set. As before, the action of $S O(2)$ reduces this to 5dimensions.

Summarizing, for fixed $0 \leq t \leq 1 / \sqrt{8}$, the solution set is always at most 5 dimensional. Varying $t$ now gives (18).

Next we consider $\mathbf{I I}_{1}$ in the splitting (19). Given a full minimal immersion $f: S^{3} \rightarrow S_{V}$ of type II, if $f$ is linearly nonrigid, that is $\operatorname{dim} \mathcal{M}_{f} \geq 1$, then the points on $\partial \mathcal{M}_{f}$ correspond to type I minimal immersions so that $\partial \mathcal{M}_{f} \subset \mathbf{I}$. Thus, to describe $\mathbf{I I}_{1}$ we consider line segments connecting pairs of points in $\mathbf{I}$ and use the connecting lemma to make sure that the points in the interior of the segment 
correspond to type II quartic minimal immersions. Since $\mathbf{I}$ is a single orbit, we may assume that one endpoint of the segment is $\langle\mathcal{I}\rangle$. We now choose $g=a+b j \in S U(2)$ so that $\gamma g \gamma \in S U(2)^{\prime}$ and let the other endpoint be $\langle\mathcal{I} \circ(\gamma g \gamma)\rangle$. By the connecting lemma, the space of components of any quartic minimal immersion corresponding to the interior of the segment connecting these two points is the $S U(2)$-module

$$
V_{\mathcal{I} \circ(\gamma g \gamma)}+V_{\mathcal{I}}
$$

and, assuming that the endpoints are distinct, the interior points correspond to type II or type III according as this is $3 R_{4}$ or $4 R_{4}$. To simplify the computations, we consider the quotient

$$
\left(V_{\mathcal{I} \circ(\gamma g \gamma)}+V_{\mathcal{I}}\right) / V_{\mathcal{I}}=V_{\mathcal{I} \circ(\gamma g \gamma)} /\left(V_{\mathcal{I} \circ(\gamma g \gamma)} \bigcap V_{\mathcal{I}}\right) .
$$

This quotient is trivial iff

$$
\langle\mathcal{I} \circ(\gamma g \gamma)\rangle=\langle\mathcal{I}\rangle,
$$

a task we also have to carry out to prove Theorem 6 since $\gamma g \gamma$ then belongs to the isotropy group of $S U(2)^{\prime}$. The quotient is equal to $R_{4}$ or $2 R_{4}$ according to whether we have type II or type III in the aforementioned segment. Technically speaking, we need to make the substitution $z \mapsto a z+b \bar{w}$ and $w \mapsto-b \bar{z}+a w$ corresponding to $\gamma g \gamma, g=a+b j$, in each of the polynomials in

$$
\begin{aligned}
& V_{\mathcal{I}}=\operatorname{span}\left\{z^{4}-\bar{w}^{4}, z^{2} \bar{w}^{2}, z^{3} w+\bar{z} \bar{w}^{3}, z \bar{z}^{2} w-\bar{z} w^{2} \bar{w}\right. \\
& \left.\quad \Im\left(z^{2} w^{2}\right),|z|^{4}-4|z|^{2}|w|^{2}+|w|^{4}\right\}
\end{aligned}
$$

and work out the components modulo $V_{\mathcal{I}}$. Elementary computations now give that $V_{\mathcal{I} \circ(\gamma g \gamma)}$ modulo $V_{\mathcal{I}}$ is spanned by the following polynomials:

$$
\begin{aligned}
& \mu z^{4}+4 \beta z^{3} \bar{w}+4 \bar{\beta} z \bar{w}^{3} \\
& \nu z^{4}-2 \alpha z^{3} \bar{w}+2 \bar{\alpha} z \bar{w}^{3} \\
& \mu z^{3} w-\beta z^{3} \bar{z}+\bar{\beta} w \bar{w}^{3}+3 \beta z^{2} w \bar{w}-3 \bar{\beta} z \bar{z} \bar{w}^{2} \\
& -2 \nu z^{3} w-\alpha z^{3} \bar{z}-\bar{\alpha} w \bar{w}^{3}+3 \alpha z^{2} w \bar{w}+3 \bar{\alpha} z \bar{z} \bar{w}^{2} \\
& \Im\left(\mu z^{2} w^{2}\right)+4 \Im\left(\beta\left(z w^{2} \bar{w}-z^{2} \bar{z} w\right)\right) \\
& \nu \Re\left(z^{2} w^{2}\right)+2 \Re\left(\alpha\left(z^{2} \bar{z} w-z w^{2} \bar{w}\right)\right),
\end{aligned}
$$

where

$$
\begin{aligned}
\alpha & =a \bar{b}\left(|a|^{2}-|b|^{2}\right) \\
\beta & =a^{3} b+\bar{a} \bar{b}^{3} \\
\mu & =a^{4}-\bar{a}^{4}+b^{4}-\bar{b}^{4} \\
\nu & =a^{2} \bar{b}^{2}+\bar{a}^{2} b^{2} .
\end{aligned}
$$


Lemma. (24) - (29) are linearly dependent iff

$$
\Re(\alpha \bar{\beta})=0 \quad \text { and } \quad \alpha \mu+2 \beta \nu=0 .
$$

Proof. We first observe that (24)-(25), (26)-(27) and (28)-(29) are mutually orthogonal. Thus, we need to study linear dependence of each pair of polynomials. The proposition now follows by case-by-case verification by splitting the first two pairs of polynomials into real and imaginary parts and evaluating each $4 \times 4$ subdeterminant of the corresponding $4 \times 6$-matrices. The last pair gives only $2 \times 2$-subdeterminants of a $2 \times 4$-matrix.

The remaining task is to work out (30) in terms of $a$ and $b$. The first equation in (30) gives

$$
\left(|a|^{2}-|b|^{2}\right) \Re\left(a^{2} b^{2}\right)=0 .
$$

It is convenient to use 'isoparametric' coordinates on $S^{3}$, that is to set

$$
a=\cos t e^{i \theta} \quad \text { and } \quad b=\sin t e^{i \phi} .
$$

$t=0, \pi / 2$ correspond to the two great orthogonal circles cut out from $S^{3}$ by the span of the first and last two coordinate axes; a fixed $0<t<\pi / 2$ corresponds to the Clifford torus $T_{t}$ parametrized by $\theta$ and $\phi$.

Case I Let $|a|^{2}=|b|^{2}$. We are on the 'middle' Clifford torus $T_{\pi / 4}$. We have $\alpha=0$ so that, the second equation in (30) reduces to $\beta \nu=0$.

If $\beta=0$ then, substituting (32) into the expression of $\beta$ we obtain

$$
\phi=-\theta+(2 k+1) \pi / 4, \quad k \in \mathbf{Z},
$$

or equivalently,

$$
a=(1 / \sqrt{2}) e^{i \theta}, \quad \text { and } \quad b=(1 / \sqrt{2}) e^{-i \theta} \epsilon^{2 k+1}, \quad k \in \mathbf{Z},
$$

where $\epsilon=e^{i \pi / 4}$.

If $\nu=0$, we get

$$
\phi=\theta+(2 k+1) \pi / 4, \quad k \in \mathbf{Z},
$$

so that

$$
a=(1 / \sqrt{2}) e^{i \theta} \quad \text { and } \quad b=(1 / \sqrt{2}) e^{i \theta} \epsilon^{2 k+1}, \quad k \in \mathbf{Z} .
$$

Summarizing Case I, the solution set is the union of 8 closed curves in $T_{\pi / 4}$ and they lift to $[0,2 \pi]^{2}$ to give straight segments with slope \pm 1 and $\theta$ - and $\phi$-intercepts being any odd multiples of $\pi / 4$.

Case II We assume that $t \neq \pi / 4$. If $t=0$ then

$$
a=e^{i \theta} \quad \text { and } \quad b=0 .
$$


We obtain the entire great circle $T_{0}$.

If $t=\pi / 2$ then

$$
a=0 \quad \text { and } \quad b=e^{i \phi}
$$

and the solution set is $T_{\pi / 2}$.

Finally, let $0<t<\pi / 2$ and $t \neq \pi / 4$. Working out each coefficient $\alpha, \beta, \mu$, $\nu$ and substituting them into the second equation in (30), we finally arrive at the solution set

$$
a=\cos t \epsilon^{l} \quad \text { and } \quad b=\sin t \epsilon^{l} \epsilon^{2 k+1}, \quad k, l \in \mathbf{Z} .
$$

For fixed $t$ as above, this is the union of 32 points and on $[0,2 \pi]^{2}$ they correspond to the intersection points of the straight segments obtained above. As $t$ moves, these points sweep 32 curves that, on $T_{\pi / 4}$, meet the existing solution set in triple intersection points and on $T_{0}$ and $T_{\pi / 2}$ they also produce 8 triple intersection points distributed equidistantly. Summarizing, the solution set consists of 48 closed curves meeting in 48 triple intersection points. Looking at each case separately, we see that the triple intersection points are given (as quaternions) by

$$
(1 / \sqrt{2})\left(\epsilon^{k}+\epsilon^{l} j\right), \quad k \not \equiv l(\bmod 2) ; \epsilon^{k}, \epsilon^{l} j, \quad k, l \in \mathbf{Z} .
$$

These form a group of order 48 and is conjugate in $S^{3}$ to the binary octahedral group $\mathbf{O}^{*}$. By abuse of notation, we denote this conjugate by the same symbol. We obtain that the orbit $\mathbf{I}$ is the 'octahedral manifold' $S^{3} / \mathbf{O}^{*}$ in $\mathcal{B}$ and the second part of Theorem 6 follows.

Looking now back at the 48 curves above, we see that on the quotient $\mathbf{I}=$ $S^{3} / \mathbf{O}^{*}$ they give exactly 3 closed curves intersecting at $\langle\mathcal{I}\rangle$. After conjugation with $\gamma$, they are orbits of the (mutually orthogonal) 1-parameter subgroups corresponding to $Z,(1 / \sqrt{2})(Y+X)$ and $(1 / \sqrt{2})(Y-X)$ in $s u(2)$, where

$$
Z=\left[\begin{array}{cc}
i & 0 \\
0 & -i
\end{array}\right], \quad X=\left[\begin{array}{cc}
0 & 1 \\
-1 & 0
\end{array}\right], \quad Y=\left[\begin{array}{ll}
0 & i \\
i & 0
\end{array}\right]
$$

form the standard orthonormal basis in $s u(2)$. Denote these orbits by $\sigma, \sigma^{\prime}$ and $\sigma^{\prime \prime}$. More explicitly, $\sigma$ is parametrized by

$$
\theta \mapsto \gamma e^{i \theta} \gamma \cdot\langle\mathcal{I}\rangle, \quad \theta \in \mathbf{R}
$$

(corresponding to $t=0$ in Case II) and $\sigma^{\prime}$ (resp. $\sigma^{\prime \prime}$ ) are parametrized by (33) with $k=l=0$ (resp. $k=1$ and $l=0)$. Note that they intersect orthogonally at $\langle\mathcal{I}\rangle$.

We now take a closer look at $\sigma$. A quick check of Case II reveals that $V_{\mathcal{I} \circ\left(\gamma e^{i \theta} \gamma\right)}$ modulo $V_{\mathcal{I}}$ does not depend on $\theta$. The same is true for

$$
V_{\mathcal{I} \circ\left(\gamma e^{i \theta} \gamma\right)}+V_{\mathcal{I}}
$$


so that the connecting lemma implies that $\sigma$ is on the boundary of the relative moduli space corresponding to any interior point of any segment connecting two distinct points of $\sigma$. We choose the midpoint of the segment connecting $\langle\mathcal{I}\rangle$ and $e^{i \pi / 8} \cdot\langle\mathcal{I}\rangle$ that has the type II representative $\mathcal{J}: S^{3} \rightarrow S^{14}$ given by

$$
\begin{aligned}
& \mathcal{J}(z, w)=(1 / \sqrt{2})\left(z^{4}, w^{4}, 2 \sqrt{3} z^{2} \bar{w}^{2}, 2 z^{3} w, 2 z w^{3}\right. \\
& \left.2 \sqrt{3}\left(z \bar{z}^{2} w-\bar{z} w^{2} \bar{w}\right), \sqrt{6} z^{2} w^{2},|z|^{4}-4|z|^{2}|w|^{2}+|w|^{4}\right) .
\end{aligned}
$$

Thus, we have $\sigma \subset \partial \mathcal{M}_{\mathcal{J}}$. Our immediate purpose is to show that $\sigma=\partial \mathcal{M}_{\mathcal{J}}$ holds. To prove this, we make a slight detour and, observing that $\mathcal{J}$ is $U(2)$ equivariant, we claim that the segment

$$
\mathbf{R} \cdot\langle\mathcal{J}\rangle \bigcap\left(\mathcal{M}^{4}\right)^{S U(2)}
$$

parametrizes all full quartic $U(2)$ equivariant minimal immersions $f: S^{3} \rightarrow S_{V}$. Indeed, the $U(2)$ equivariant quartic minimal immersions are parametrized by the fixed point set $\left(\mathcal{M}^{4}\right)^{U(2)}$ so that the claim follows once we show that $\left(R_{8}^{\prime}\right)^{U(2)}$ is 1-dimensional. Since $R_{8}^{\prime}$ is $S U(2)$ fixed, we have $\left(R_{8}^{\prime}\right)^{U(2)}=\left(R_{8}^{\prime}\right)^{\Gamma}$, where

$$
\Gamma=\left\{\operatorname{diag}\left(e^{i \theta}, e^{i \theta}\right) \mid \theta \in \mathbf{R}\right\} \subset S U(2)^{\prime}
$$

is the center of $U(2)$. As noted above, $\gamma \in O(4)$ switches $R_{8}^{\prime}$ and $R_{8}$ and $\Gamma$ to

$$
\Gamma^{\prime}=\left\{\operatorname{diag}\left(e^{i \theta}, e^{-i \theta}\right) \mid \theta \in \mathbf{R}\right\}
$$

the standard (1-dimensional) maximal torus in $S U(2)$. Thus, $\left(R_{8}^{\prime}\right)^{\Gamma}$ corresponds to $\left(R_{8}\right)^{\Gamma^{\prime}}$. On the other hand, $\Gamma^{\prime}$ acts on the standard basis in $R_{8}$ diagonally with a unique $\Gamma^{\prime}$-fixed polynomial $-z^{2} w^{2}$ and the claim follows.

Remark. For $p$ even, $W_{p}=\mathcal{H}_{2}^{p / 2}$, where the $S U(2)$-module structure on the space of spherical harmonics on $S^{2}$ is given by the projection $S U(2) \rightarrow S O(3)$. Thus we also have $R_{p}=\mathcal{H}_{2}^{p / 2}$ as real modules. The $S U(2)^{\prime}$ orbit of $\langle J\rangle$ is $\mathbf{R} P^{2}$ embedded minimally into its respective 8 -sphere as the image of the standard minimal immersion $f_{2}: S^{2} \rightarrow S^{8}$. Indeed, $\left(\mathcal{F}^{4}\right)^{S U(2)}=R_{8}^{\prime}=\mathcal{H}_{2}^{4}$ and $\left(R_{8}^{\prime}\right)^{U(2)}$ corresponds to the zonals $\left(\mathcal{H}_{2}^{4}\right)^{S O(2)}$ whose $S O(3)$ orbit on the unit sphere gives the image of $f_{2}$.

We are now ready to prove that $\sigma=\partial \mathcal{M}_{\mathcal{J}}$. By the above, $\sigma \subset \partial \mathcal{M}_{\mathcal{J}}$ so that $\mathcal{M}_{\mathcal{J}}$ is at least 2-dimensional. Since $\langle\mathcal{J}\rangle$ is $\Gamma$-fixed, $\Gamma$ leaves $\mathcal{M}_{\mathcal{J}}$ and its boundary invariant. $\Gamma$ acts on $\partial \mathcal{M}_{\mathcal{J}}$ without fixed points since a fixed point is automatically $U(2)$ fixed and there are only two of these on the entire boundary. Thus, $\operatorname{dim} \mathcal{M}_{\mathcal{J}}$ must be even, therefore 2 or 4 . Finally, it cannot be 4 since, in that case, $\partial \mathcal{M}_{\mathcal{J}}$ 
would be a topological $S^{3}$ (by convexity) and it would have to coincide with $\mathbf{I}$ (for reasons of dimension). The latter is $S^{3} / \mathbf{O}^{*}$ that is topologically distinct from $S^{3}$. We obtain that $\mathcal{M}_{\mathcal{J}}$ is a flat 2 -disk $\mathcal{D}$ with center $\langle\mathcal{J}\rangle$, in particular, $\partial \mathcal{M}_{\mathcal{J}}$ is 1-dimensional, and thus it must coincide with $\sigma$.

The argument is entirely analogous for $\sigma^{\prime}$ and $\sigma^{\prime \prime}$ so that they are the boundary circles of 2-disks $\mathcal{D}^{\prime}$ and $\mathcal{D}^{\prime \prime}$. Note that $\mathcal{D}, \mathcal{D}^{\prime}$ and $\mathcal{D}^{\prime \prime}$ are orthogonal to each other at the common boundary point $\langle\mathcal{I}\rangle$. We now let $S U(2)^{\prime}$ act on this configuration and realize that $\mathcal{D}^{\prime}$ and $\mathcal{D}^{\prime \prime}$ are on the $S U(2)^{\prime}$ orbit of $\mathcal{D}$. We thus arrive at (21).

At this point, without having a detailed study of the type III quartic minimal immersions, we can only assert that $\mathbf{I I}_{0}$ is of dimension at least 3 and postpone the proof of (20). More specifically, we claim now that the antipodal orbit

$$
S U(2)^{\prime} \cdot\left\langle\mathcal{I}_{o}\right\rangle
$$

consists of type II linearly rigid quartic minimal immersions. To do this, we first determine the antipodal of $\mathcal{I}$. Recall that $\mathcal{I}$ has orthogonal components with the same norm so that the antipodal lemma applies. It immediately gives that the antipodal $\mathcal{I}_{o}$ is of type II. The $S U(2)^{\prime}$-orbit through $\left\langle\mathcal{I}_{o}\right\rangle$, being the antipodal of $\mathbf{I}$, is again an octahedral manifold. It remains to show that $\mathcal{I}_{o}$ is linearly rigid. Assume that $\mathcal{M}_{\mathcal{I}_{o}}$ is nontrivial and consider a line segment through $\left\langle\mathcal{I}_{o}\right\rangle$ with endpoints $\left\langle f_{1}\right\rangle$ and $\left\langle f_{2}\right\rangle$ on $\partial \mathcal{M}_{\mathcal{I}_{o}}$. Clearly, $f_{1}$ and $f_{2}$ are of type I. Consequently, the antipodals $f_{1}^{o}$ and $f_{2}^{o}$ are of type II. Let $\langle f\rangle$ be the intersection of the segment connecting $\left\langle f_{1}^{o}\right\rangle$ and $\left\langle f_{2}^{o}\right\rangle$ with the line $\mathbf{R} \cdot\left\langle\mathcal{I}_{o}\right\rangle$. We claim that $f$ is of type III which is a contradiction since, in this case, it should be congruent to $\mathcal{I}$ (the antipodal of $\mathcal{I}_{o}$ ) that is of type I. To prove the claim we use the connecting lemma and compute

$$
\begin{aligned}
V_{f} & =V_{f_{1}^{o}}+V_{f_{2}^{o}} \\
& =V_{f_{1}}^{\perp}+V_{f_{2}}^{\perp} \\
& =\left(V_{f_{1}} \bigcap V_{f_{2}}\right)^{\perp} .
\end{aligned}
$$

On the other hand

$$
\begin{aligned}
\operatorname{dim}\left(V_{f_{1}} \bigcap V_{f_{2}}\right) & =\operatorname{dim} V_{f_{1}}+\operatorname{dim} V_{f_{2}} \\
& -\operatorname{dim}\left(V_{f_{1}}+V_{f_{2}}\right) \\
& =10+10-\operatorname{dim} V_{\mathcal{I}_{o}} \\
& =10+10-(25-10)=5 .
\end{aligned}
$$

Theorem 7 follows (with the proof of (20) postponed).

We now consider type III quartic minimal immersions, and claim that the antipodal $\mathcal{J}_{o}$ of $\mathcal{J}$ is of type III. These are actually the two endpoints of the segment (35) parametrizing the $U(2)$ equivariant quartic minimal immersions. Recall that 
$\langle\mathcal{J}\rangle$ is the midpoint of the segment connecting $\langle\mathcal{I}\rangle$ and $\left\langle\mathcal{I} \circ\left(\gamma e^{i \pi / 8} \gamma\right)\right\rangle$ both of type I. Thus, the antipodal of $\langle\mathcal{J}\rangle$ must be on the segment connecting $\left\langle\mathcal{I}_{o}\right\rangle$ and $\left\langle\mathcal{I} \circ\left(\gamma e^{i \pi / 8} \gamma\right)_{o}\right\rangle$ provided that this segment is on the boundary $\mathcal{B}$. Thus, by the connecting lemma again, we need to work out

$$
V_{\mathcal{I}_{o}}+V_{\mathcal{I} \circ\left(\gamma e^{i \pi / 8} \gamma\right)_{o}}
$$

By the antipodal lemma, this is equal to

$$
V_{\mathcal{I}}^{\perp}+V_{\mathcal{I} \circ\left(\gamma e^{i \pi / 8} \gamma\right)}^{\perp}=\left(V_{\mathcal{I}} \bigcap V_{\mathcal{I} \circ\left(\gamma e^{i \pi / 8} \gamma\right)}\right)^{\perp}
$$

On the other hand

$$
\operatorname{dim}\left(V_{\mathcal{I}} \bigcap V_{\mathcal{I} \circ\left(\gamma e^{i \pi / 8} \gamma\right)}\right)=\operatorname{dim} V_{\mathcal{I}}+\operatorname{dim} V_{\mathcal{I} \circ\left(\gamma e^{i \pi / 8} \gamma\right)}-\operatorname{dim}\left(V_{\mathcal{I}}+V_{\mathcal{I} \circ\left(\gamma e^{i \pi / 8} \gamma\right)}\right)
$$

and this is $10+10-15=5$-dimensional and the claim follows.

Lemma. Let $f: S^{3} \rightarrow S_{V}$ be a full $S U(2)$ equivariant quartic minimal immersion of type III. Then, we have

$$
\operatorname{dim} \mathcal{M}_{f} \geq 4
$$

Proof. As before, the Lie algebra $s u(2)$ is considered as the tangent space of $S^{3}$ at the identity. For $U \in s u(2)$, we denote by $\tilde{U}$, the right invariant extension of $U$ on $S^{3}$. Given $C \in S^{2} V$, we define the linear map

$$
\Psi(C): s u(2) \times s u(2) \rightarrow \mathcal{P}^{8},
$$

by

$$
\Psi(C)\left(U, U^{\prime}\right)=\left\langle C f_{*}(\tilde{U})^{\sim}, f_{*}\left(\tilde{U}^{\prime}\right)^{\sim}\right\rangle=\left\langle C \tilde{U}(f), \tilde{U}^{\prime}(f)\right\rangle,
$$

where $\mathcal{P}^{q}$ is the space of homogeneous polynomials of degree $q$ on $\mathbf{R}^{4}$. Evaluating $\tilde{U}(f)$ on the basis $Z, X, Y$ it follows easily that this function belongs to $\mathcal{P}^{4}$ so that $\Psi(C)$ maps into $\mathcal{P}^{8}$. Since $\Psi(C)$ is symmetric in the arguments $U$ and $U^{\prime}$, it can be considered as a linear map

$$
\Psi(C): S^{2}(s u(2)) \rightarrow \mathcal{P}^{8},
$$

or equivalently, an element $\Psi(C) \in \mathcal{P}^{8} \otimes S^{2}(s u(2))$. We now vary $C$ in $S^{2} V$ and obtain the linear map

$$
\Psi: S^{2} V \rightarrow \mathcal{P}^{8} \otimes S^{2}(s u(2))
$$

Since the right invariant vector fields (pointwise) span each tangent space in $S^{3}$, we have

$$
\operatorname{ker} \Psi=\mathcal{F}_{f}
$$


To estimate this kernel we first observe that $\Psi$ is a homomorphism of $S U(2)$ modules, where the module structure on $V$ is given by $S U(2)$ equivariance of $f$. Explicitly, for $g \in S U(2)$, we have

$$
\Psi(g \cdot C)\left(A d(g)(U), A d(g)\left(U^{\prime}\right)\right)=\Psi(C)\left(U, U^{\prime}\right) \circ L_{g^{-1}} .
$$

Since $f$ is of type III, we have $V=4 R_{4}$ as $S U(2)$-modules. Thus

$$
\begin{aligned}
S^{2} V & =S^{2}\left(4 R_{4}\right)=4 S^{2}\left(R_{4}\right) \oplus 6\left(R_{4} \otimes R_{4}\right) \\
& =10 R_{8} \oplus 6 R_{6} \oplus 10 R_{4} \oplus 6 R_{2} \oplus 10 R_{0}
\end{aligned}
$$

(cf. (9)). On the other hand,

$$
\begin{aligned}
\mathcal{P}^{8} & =\mathcal{H}^{8} \oplus \mathcal{H}^{6} \oplus \mathcal{H}^{4} \oplus \mathcal{H}^{2} \oplus \mathcal{H}^{0} \\
& =9 R_{8} \oplus 7 R_{6} \oplus 5 R_{4} \oplus 3 R_{2} \oplus R_{0},
\end{aligned}
$$

where the first row is isomorphism as $S O(4)$-modules, the second as $S U(2)$ modules. Finally, $s u(2)=R_{2}$ so that

$$
S^{2}(s u(2))=R_{4} \oplus R_{0} .
$$

Putting all these together, we obtain

$$
\mathcal{P}^{8} \otimes S^{2}(s u(2))=9 R_{12} \oplus 16 R_{10} \oplus 30 R_{8} \oplus 31 R_{6} \oplus 30 R_{4} \oplus 18 R_{2} \oplus 6 R_{0} .
$$

Comparing this with the domain of $\Psi$ we see that $4 R_{0}$ must be in the kernel.

Let III denote the set of points that correspond to type III quartic minimal immersions. Recall also that $\mathcal{J}_{o}$ is the unique full $U(2)$ equivariant quartic boundary minimal immersion of type III.

Theorem 8. III is everywhere dense, open and connected in the 8-dimensional boundary $\mathcal{B}$. $\mathcal{M}_{\mathcal{J}_{o}} \subset \mathcal{B}$ is 6 -dimensional and

$$
\mathbf{I I I}=S U(2)^{\prime} \cdot \operatorname{int} \mathcal{M}_{\mathcal{J}_{o}} .
$$

Proof. First of all, by (18), the complement of $\mathbf{I I I}$ in $\mathcal{B}$ is of codimension 2 so that III is everywhere dense, open and connected in $\mathcal{B}$. We now claim that

$$
\operatorname{dim} \mathcal{M}_{\mathcal{J}_{o}}=6 .
$$

$U(2)$ leaves $\mathcal{M}_{\mathcal{J}_{o}}$ invariant since it fixes $\left\langle\mathcal{J}_{o}\right\rangle$. By local unicity of the $U(2)$ fixed points, the center $\Gamma$ of $U(2)$ acts on $\partial \mathcal{M}_{\mathcal{J}_{o}}$ without fixed points. It follows that $\operatorname{dim} \mathcal{M}_{\mathcal{J}_{o}}$ is even. By the previous lemma, $\operatorname{dim} \mathcal{M}_{\mathcal{J}_{o}}$ is either 4 or 6 . The claim will follow if we show that $\operatorname{dim} \mathcal{M}_{\mathcal{J}_{o}} \geq 6$. 
Consider the $S U(2)$-module homomorphism $\Psi: S^{2} V \rightarrow \mathcal{P}^{8} \otimes S^{2}(s u(2))$ introduced in the previous lemma for $f=\mathcal{J}_{o}: S^{3} \rightarrow S_{V}$. Since $\mathcal{J}_{o}$ is $U(2)$ equivariant, $\Psi$ is a homomorphism of $U(2)$-modules, where the $U(2)$-module structure on $V$ is given by the equivariance of $\mathcal{J}_{o}$, and the center $\Gamma \subset U(2)$ acts on $s u(2)$ trivially. Being a $U(2)$-submodule of $\mathcal{H}^{4}, V$ (complexified) decomposes according to (10). We have

$$
V \otimes_{\mathbf{R}} \mathbf{C}=\mathcal{H}^{4,0} \oplus \mathcal{H}^{3,1} \oplus \mathcal{H}^{1,3} \oplus \mathcal{H}^{0,4}
$$

Indeed, since $\operatorname{dim} V=20$, we need only to show that $\mathcal{H}^{2,2}$ is not a component of $V$. This, however, follows from the antipodal and connecting lemmas. Indeed, $\mathcal{I}$ and $\mathcal{I} \circ\left(\gamma e^{i \pi / 8} \gamma\right)$ do have components in $\mathcal{H}^{2,2}$, and so does $\mathcal{J}$ since $\langle\mathcal{J}\rangle$ is the midpoint of the segment connecting $\langle\mathcal{I}\rangle$ and $\left\langle\mathcal{I} \circ\left(\gamma e^{i \pi / 8} \gamma\right)\right\rangle$. Since $\mathcal{I}$ has orthogonal components with the same norm the antipodal lemma applies, and we see that $\mathcal{I}_{o}$ and $\mathcal{I} \circ\left(\gamma e^{i \pi / 8} \gamma\right)_{o}$ do not have components in $\mathcal{H}^{2,2}$. The same holds for $\mathcal{J}_{o}$ as $\left\langle\mathcal{J}_{o}\right\rangle$ is the midpoint of the segment connecting $\left\langle\mathcal{I}_{o}\right\rangle$ and $\left\langle\mathcal{I} \circ\left(\gamma e^{i \pi / 8} \gamma\right)_{o}\right\rangle$.

As in the previous lemma, we now count the trivial components in the $U(2)$ modules that contribute to the domain and range of the (complexified) $\Psi$. Since $\Gamma$ acts on these components as a character, we will also keep track of the corresponding weights. $S^{2}(V)$ contains 6 trivial components with zero weight; 4 coming from the symmetric squares of the components in (38) (e.g. $S^{2}\left(\mathcal{H}^{3,1}\right)$ is contained in $\mathcal{H}^{3,1} \otimes \mathcal{H}^{1,3}$ that has weight $\left.(3-1)+(1-3)=0\right)$, and 2 coming from $\mathcal{H}^{4,0} \otimes \mathcal{H}^{0,4}$ and $\mathcal{H}^{3,1} \otimes \mathcal{H}^{1,3}$. In addition, $S^{2}(V)$ contains one trivial component for each weight $6,2,-2,-6$, (e.g. $\mathcal{H}^{4,0} \otimes \mathcal{H}^{3,1}$ has weight $(4-0)+(3-1)=6$ ). As $U(2)$-modules, $S^{2}(s u(2))=\mathcal{H}^{2,2} \oplus \mathcal{H}^{0}$ since $\Gamma$ acts on $s u(2)$ trivially. All the trivial components in $\mathcal{P}^{8} \otimes S^{2}(s u(2))$ are in $\mathcal{H}^{4} \otimes\left(\mathcal{H}^{2,2} \oplus \mathcal{H}^{0}\right)$, and, by (10), we have 2 trivial components with zero weight, and one trivial component for each weight $4,2,-2,-4$. Comparing, we see that the kernel of $\Psi$ must contain at least 4 trivial components with zero weight, and two trivial components with weights \pm 6 . We see that ker $\Psi$ is at least 6 -dimensional. The claim follows.

By the transversality lemma, the $S U(2)^{\prime}$ orbit of $\operatorname{int} \mathcal{M}_{\mathcal{J}_{o}}$ is an 8-dimensional smooth manifold since $\Gamma$ leaves $\mathcal{M}_{\mathcal{J}_{o}}$ invariant, and $S U(2)^{\prime}$ does not have 2dimensional subgroups. This orbit must then be open in $\mathcal{B}$. Its boundary is contained in $\mathbf{I} \cup \mathbf{I I}$. It follows that $S U(2)^{\prime} \cdot \operatorname{int} \mathcal{M}_{\mathcal{J}_{o}}$ is a connected component of III (and its boundary is equal to I $\cup \mathbf{I I}$ ). Since III is connected, Theorem 8 follows. As a byproduct, we obtain that $\mathbf{I I}$ and $\mathbf{I I}_{0}$ are connected (and 6 -dimensional) since $\mathbf{I} \subset \mathbf{I I}$ and $\mathbf{I I}_{1} \subset \mathbf{I I}$ are of codimension $\geq 2$.

\section{References}

[1] E. Calabi, Minimal immersions of surfaces in euclidean spheres, J. Diff. Geom. 1 (1967), 111-125.

[2] D. DeTurck and W. Ziller, Minimal isometric immersions of spherical space forms in spheres, Comment. Math. Helvetici 67 (1992), 428-458. 
[3] M. DoCarmo and N. Wallach, Minimal immersions of spheres into spheres, Ann. of Math. 93 (1971), 43-62.

[4] C. M. Escher, Minimal isometric immersions of inhomogeneous spherical space forms into spheres - A necessary condition for existence, Trans. Amer. Math. Soc. 348(9) (1996), 3713-3732.

[5] H. Gauchman and G. Toth, Fine structure of the space of spherical minimal immersions, Trans. Amer. Math. Soc. 348(6) (1996), 2441-2463.

[6] K. Mashimo, Minimal immersions of 3-dimensional spheres into spheres, Osaka J. Math. 2 (1984), 721-732.

[7] K. Mashimo, Homogeneous totally real submanifolds in $S^{6}$, Tsukuba J. Math. 9 (1985), 185-202.

[8] J. D. Moore, Isometric immersions of space forms into space forms, Pacific J. Math. 40 (1976), 157-166.

[9] Y. Muto, The space $W_{2}$ of isometric minimal immersions of the three-dimensional sphere into spheres, Tokyo J. Math. 7 (1984), 337-358.

[10] R. T. Smith, Harmonic mappings of spheres, Thesis, Warwick University (1980).

[11] T. Takahashi, Minimal immersions of Riemannian manifolds, J. Math. Soc. Japan 18 (1966), 380-385.

[12] G. Toth, Harmonic Maps and Minimal Immersions through Representation Theory, Academic Press, Boston 1990.

[13] G. Toth, Classification of quadratic harmonic maps of $S^{3}$ into spheres, Indiana Univ. Math. J. 36(2) (1987), 231-239.

[14] G. Toth, Eigenmaps and the space of minimal immersions between spheres, Indiana Univ. Math. J. 46(2) (1997), 637-658.

[15] G. Toth, Universal constraints on the range of eigenmaps and spherical minimal immersions, to appear in Trans. Amer. Math. Soc.

[16] N. I. Vilenkin, Special Functions and the Theory of Group Representations, AMS Translations of Mathematical Monographs, 22, 1968.

[17] N. Wallach, Minimal immersions of symmetric spaces into spheres. In Symmetric Spaces, Dekker, New York 1972, pp. 1-40.

[18] M. Wang and W. Ziller, On isotropy irreducible Riemannian manifolds, Acta Math. 166 (1991), 223-261.

Gabor Toth

Department of Mathematics

Rutgers University

Camden, New Jersey 08102

USA

e-mail: gtoth@crab.rutgers.edu
Wolgang Ziller

Department of Mathematics

University of Pennsylvania

Philadelphia, Pennsylvania 19104

USA

e-mail: wziller@math.upenn.edu

(Received: November 12, 1997) 